\title{
Automatic differentiation for Fourier series and the radii polynomial approach
}

\author{
Jean-Philippe Lessard* $\quad$ J. D. Mireles James ${ }^{\dagger} \quad$ Julian Ransford ${ }^{\ddagger}$
}

\begin{abstract}
In this work we develop a computer-assisted technique for proving existence of periodic solutions of nonlinear differential equations with non-polynomial nonlinearities. We exploit ideas from the theory of automatic differentiation in order to formulate an augmented polynomial system. We compute a numerical Fourier expansion of the periodic orbit for the augmented system, and prove the existence of a true solution nearby using an a-posteriori validation scheme (the radii polynomial approach). The problems considered here are given in terms of locally analytic vector fields (i.e. the field is analytic in a neighborhood of the periodic orbit) hence the computer-assisted proofs are formulated in a Banach space of sequences satisfying a geometric decay condition. In order to illustrate the use and utility of these ideas we implement a number of computer-assisted existence proofs for periodic orbits of the Planar Circular Restricted Three-Body Problem (PCRTBP).
\end{abstract}

Key words. Rigorous numerics, automatic differentiation, Fourier series, Contraction Mapping Theorem, periodic solutions

\section{Introduction}

Questions concerning the existence of periodic motions are at the heart of the qualitative theory of nonlinear differential equations. When a system is neither a perturbation nor undergoing a Hopf bifurcation, these questions have a global flavor and may be difficult to resolve via classical pen and paper arguments. Numerical simulations provide insight, and in particular may suggest the existence of periodic orbits. In recent years considerable effort has gone into the development of mathematically rigorous a-posteriori methods which close the gap between numerical experiment and mathematical proof.

The present work develops a computer-assisted argument for studying periodic solutions of differential equations with non-polynomial nonlinearities given by elementary functions. Our method consists of formulating a certain fixed point problem on a Banach space of Fourier coefficients, and enables us to obtain quantitative information about analytic properties of the solution. More precisely we study analytic differential equations and obtain analytic parameterizations of periodic orbits in terms of Fourier series, bounds on the decay rate of the coefficients in the tail, lower bounds on the size of the domain of analyticity, and bounds on derivatives of the solution.

In order to implement our computer-assisted argument we borrow an idea from the theory of automatic differentiation. The point is that we must efficiently compose an unknown Fourier series with a non-polynomial vector field. We use "automatic differentiation for Fourier series" to transform the given problem into a polynomial problem in a larger number of variables. The transformed problem is amenable to existing methods of computer-assisted analysis, and we employ the techniques discussed in [1] to complete the argument.

In Section 2.6 we illustrate the utility of the proposed method and give a number of validated periodic orbits for a classical problem of celestial mechanics (namely the planar circular restricted three body problem-PCRTBP). For this problem we

\footnotetext{
*Université Laval, Département de Mathématiques et de Statistique, 1045 avenue de la Médecine, Québec, QC, G1V0A6, Canada. jean-philippe.lessard@mat.ulaval.ca. This author was partially supported by an NSERC Discovery Grant.

${ }^{\dagger}$ Florida Atlantic University, Department of Mathematical Sciences, Science Building, Room 234, 777 Glades Road, Boca Raton, Florida, 33431 , USA. jmirelesjames@fau.edu. This author was partially supported by the NSF Grant DSM - 1318172

¥Université Laval, Département de Mathématiques et de Statistique, 1045 avenue de la Médecine, Québec, QC, G1V0A6, Canada. julian.ransford.1@ulaval.ca. This author was partially supported by the NSERC program Undergraduate Student Research Awards (USRA).
} 
apply the automatic differentiation argument in order to obtain the augmented system of polynomial equations, derive the error estimates necessary to apply the method of radii polynomials, and implement the a-posteriori validation. We wish to stress that the orbits, and others similar, to those studied in Section 2.6 have been shown to exist by earlier authors using other methods (see also Section 1.3 below). The novelty of the present work is a new approach to computer-assisted proofs exploiting automatic differentiation for Fourier analysis of non-polynomial problems. We choose to work with the PCRTBP because it is a widely studied non-polynomial problem which appears in many serious applications, including a number of computer assisted studies. Before concluding the present introductory discussion, we state Theorem 1 , which provides some insight into the nature of our results.

Theorem 1. Let $x(t), y(t)$ be the trigonometric polynomials

$$
x(t)=a_{0}+2 \sum_{k=1}^{23} a_{k} \cos (k \omega t) \quad \text { and } \quad y(t)=-2 \sum_{k=1}^{23} b_{k} \sin (k \omega t),
$$

with $a_{k}, b_{k}$ the numbers given in Table 1 , and $\omega=1.0102$. Let $\gamma(t)=[x(t), y(t)]$ and $T^{*}=2 \pi / \omega$. Then there is a real analytic function $\gamma^{*}:\left[0, T^{*}\right] \rightarrow \mathbb{R}^{2}$ denoted component-wise by $\gamma^{*}=\left[\gamma_{1}^{*}, \gamma_{2}^{*}\right]$ such that

1. $\gamma^{*}$ is a $T^{*}$-periodic solution of the Planar Circular Restricted Three-Body Problem (PCRTBP) given in (2.1) with mass parameter $\mu=0.0123$. (The PCRTBP is discussed in Section 2).

2. $\gamma^{*}$ is a symmetric solution in the sense that $\gamma_{1}^{*}(t)$ is given by a cosine series and $\gamma_{2}^{*}(t)$ is given by a sine series.

3. $\gamma^{*}$ is $C^{0}$ close to $\gamma$. More precisely, for $r=9.8 \times 10^{-11}$,

$$
\sup _{t \in\left[0, T^{*}\right]}\left|\gamma_{1}^{*}(t)-x(t)\right| \leq r \quad \text { and } \quad \sup _{t \in\left[0, T^{*}\right]}\left|\gamma_{2}^{*}(t)-y(t)\right| \leq r
$$

4. The function $\gamma^{*}$ can be extended to a T-periodic analytic function on a complex strip having width at least

$$
\ln (1.14) / \omega \approx 0.1297
$$

5. The decay rates of the Fourier coefficients $a_{k}^{*}$ of $\gamma_{1}^{*}$ and $b_{k}^{*}$ of $\gamma_{2}^{*}$ satisfy the bounds

$$
\left|a_{k}^{*}\right|,\left|b_{k}^{*}\right| \leq \frac{7.4 \times 10^{-10}}{1.14^{k}}, \quad \text { for } k \geq 24
$$

The orbit itself is illustrated in Figure 4.

The remainder of the paper is organized as follows: The remaining sections of the introduction (Sections 1.1, 1.2 and 1.3) are devoted to some brief discussion of the literature pertaining to respectively the method of radii polynomials, the technique known as automatic differentiation, and distinctions between the geometric/phase space versus the functional analytic approach to computer-assisted proof in nonlinear analysis. These sections motivate the work to follow. In Sections 1.4 and 1.5, we review the basic notions of Automatic Differentiation for Taylor series and describe the situation for Fourier series. In Section 2, we begin discussing the Planar Circular Restricted Three-Body Problem, the main example of the paper. We review the equations of motions and in Section 2.1, we illustrate the Automatic Differentiation scheme for the problem. Then, in Sections 2.2 and 2.3 we develop the appropriate Banach Spaces and the associated $F(x)=0$ problem. In Section 2.4 and 2.5, we develop the Newton-like operator and the radii polynomials for the problem. Finally in Section 2.6 we present the results of a number of computer-assisted proofs. 


\begin{tabular}{|c|c|c|}
\hline$k$ & $a_{k}$ & $b_{k}$ \\
\hline 0 & $-9.768220550865100 \times 10^{-1}$ & - \\
\hline 1 & $-1.206409736493824 \times 10^{-1}$ & $-2.339364883946253 \times 10^{-1}$ \\
\hline 2 & $-1.347990876182309 \times 10^{-2}$ & $6.397869958624213 \times 10^{-3}$ \\
\hline 3 & $2.352585563377883 \times 10^{-3}$ & $-1.790561590462826 \times 10^{-3}$ \\
\hline 4 & $-5.122863209781185 \times 10^{-4}$ & $4.373987650320329 \times 10^{-4}$ \\
\hline 5 & $1.219044853784686 \times 10^{-4}$ & $-1.093231659117833 \times 10^{-4}$ \\
\hline 6 & $-3.055685576355795 \times 10^{-5}$ & $2.814155571301070 \times 10^{-5}$ \\
\hline 7 & $7.934843979226718 \times 10^{-6}$ & $-7.428978433135244 \times 10^{-6}$ \\
\hline 8 & $-2.114596180594020 \times 10^{-6}$ & $2.001931208169388 \times 10^{-6}$ \\
\hline 9 & $5.748968790488728 \times 10^{-7}$ & $-5.486299609956681 \times 10^{-7}$ \\
\hline 10 & $-1.588047255749821 \times 10^{-7}$ & $1.524601425602110 \times 10^{-7}$ \\
\hline 11 & $4.444105347947109 \times 10^{-8}$ & $-4.286470381069696 \times 10^{-8}$ \\
\hline 12 & $-1.257223241334567 \times 10^{-8}$ & $1.217145490467260 \times 10^{-8}$ \\
\hline 13 & $3.589443646509739 \times 10^{-9}$ & $-3.485577927995539 \times 10^{-9}$ \\
\hline 14 & $-1.032912424206540 \times 10^{-9}$ & $1.005555404303114 \times 10^{-9}$ \\
\hline 15 & $2.992744553664615 \times 10^{-10}$ & $-2.919684209251029 \times 10^{-10}$ \\
\hline 16 & $-8.723250537889003 \times 10^{-11}$ & $8.525779373911926 \times 10^{-11}$ \\
\hline 17 & $2.556153430692412 \times 10^{-11}$ & $-2.502217287937952 \times 10^{-11}$ \\
\hline 18 & $-7.525630237426317 \times 10^{-12}$ & $7.376943496986842 \times 10^{-12}$ \\
\hline 19 & $2.225016823097366 \times 10^{-12}$ & $-2.183690063445679 \times 10^{-12}$ \\
\hline 20 & $-6.603543558111446 \times 10^{-13}$ & $6.487830301502292 \times 10^{-13}$ \\
\hline 21 & $1.966611110890260 \times 10^{-13}$ & $-1.933996961613629 \times 10^{-13}$ \\
\hline 22 & $-5.875220903859556 \times 10^{-14}$ & $5.782732245351016 \times 10^{-14}$ \\
\hline 23 & $1.760271955315164 \times 10^{-14}$ & $-1.733885618947898 \times 10^{-14}$ \\
\hline & & \\
\hline
\end{tabular}

Table 1: The Fourier coefficients of $(x(t), y(t))$ of the inner most green periodic orbits on the left in Figure 4 . The frequency of the orbit is $\omega=1.0102$. There are 24 Fourier coefficients per component. We could prove the existence of the orbit with $\nu=1.14$ and $r=7.4 \times 10^{-10}$. We also proved the existence with $\nu=1.09$ and $r=9.8 \times 10^{-11}$.

\subsection{Computer-Assisted Proofs and the Radii Polynomial Approach}

The radii polynomial approach refers to a tool kit for a posteriori computer-assisted verification of the existence of a zero of a nonlinear operator equation

$$
F(x)=0
$$

defined on an infinite-dimensional Banach space. The solution $x$ may represent an invariant set of a dynamical system like a steady state, a periodic orbit, a connecting orbit, a stable manifold, etc. It could also be a minimizer of an action functional, an eigenpair of an eigenvalue problem or a solution to a boundary value problem. The radii polynomial approach consists of taking a finite-dimensional projection of (1.1), computing an approximate solution $\bar{x}$ (e.g. using Newton's method), constructing an approximate inverse $A$ of $D F(\bar{x})$, and then proving the existence of a fixed point for the Newton-like operator

$$
T(x) \stackrel{\text { def }}{=} x-A F(x)
$$

by applying the Contraction Mapping Theorem (CMT) on closed balls about $\bar{x}$. The hypotheses of the CMT are rigorously verified by deriving a system of polynomial equations (the radii polynomials) whose coefficients carry the relevant information about the nonlinear mapping (1.2), the topology of the solution space, the given numerical approximate solution $\bar{x}$, and the choice of approximate inverse $A$ for the derivative of the mapping $F$. The question "Is $T$ a contraction on some neighborhood of $\bar{x}$ ?" is reduced to a question about the sign of some polynomials. By studying the maximum interval on which these polynomials are negative we obtain bounds on the size of both the smallest and largest neighborhoods on which $T$ is a contraction. These give rise respectively to the best computer-assisted error and isolation bounds which can be obtained relative to the choices defining $T$. We note that while error bounds are of most interest, the isolation bounds are also useful in some validated continuation arguments $[2,3]$.

Before proceeding any further, we hasten to mention the existence of several other computer-assisted methods based on the fixed points theorems, predating the radii polynomial approach, designed to solve functional equations. For instance, 
rigorous computational methods based on the Newton-Kantorovich theorem [4] have been commun place for a while (e.g. see [5] and the references therein), and the approach due to Yamamoto [6] and the infinite-dimensional Krawczyk operator [7] are also closely related approaches.

The radii polynomial approach has been applied to a host of problems in differential equations/dynamical systems theory including the study of initial value problems [8], equilibrium solutions of partial differential equations [2,9, 10], periodic solutions of ordinary, delay and partial differential equations $[1,11,12,13]$, stable/unstable invariant manifolds for differential equations [14], solutions of boundary value problems [15], eigenvalue/eigenfunction problems [16], connecting orbit problems for differential equations [17, 18], and standing wave patterns [19].

One possible criticism of this collection of methods is that it appears, at first glance, limited to problems with polynomial nonlinearities. Indeed, up to now, this approach has been applied only to problems with quadratic and cubic nonlinearities. The present work puts to rest this criticism and illustrates the wider applicability of these methods.

\subsection{Non-Polynomial Nonlinearities: Automatic Differentiation, Spectral Methods, and Computer-Assisted Proof}

The need to efficiently compute the composition of a polynomial with an elementary function comes up in many numerical analysis applications, and research into algorithms for efficient manipulation of polynomials remains an active area. A classical discussion of semi-numerical algorithms for computing polynomial expansions of $e^{P}, \sin (P), P^{k}$, etc, with $P$ polynomial appears in the book of Knuth [20]. These techniques are nowadays collectively referred to as "automatic differentiation." We note that automatic differentiation as a tool in effective numerical integration schemes appears in the literature as early as [21].

The key to the automatic differentiation of $f(P)$ when $P$ is a polynomial and $f$ is an "elementary functions of mathematical physics" (trigonometric functions, Bessel functions, elliptic functions, etc) is that these $f$ themselves arise as solutions of some systems of linear differential equations. Power matching schemes applied to these differential equations reduce the cost of computing $f(P)$ to the cost of multiplying polynomials. A survey of the AD literature is beyond the scope of the present work and in addition to the works just cited we refer the interested reader to $[22,23,24]$ for more complete exposition.

$\mathrm{AD}$ tools are used extensively for computer-assisted proofs in nonlinear analysis, especially for problems involving Taylor series expansions. Chapters 4 and 5 of the book by Tucker [25] contain many examples and additional references to the literature. The reader may also find helpful the discussion in [23]. A number of software packages and libraries for computer-assisted proof in dynamical systems theory employ AD tools for Taylor series. See for example [26, 27, 28] for discussion of the COSY software package, $[29,30,31]$ for discussion of the CAPD libraries, and [32] for the INTLAB library for MATLAB.

Similar semi-numerical algorithms exist which evaluate compositions in bases other than Taylor. For example, the case of Fourier series is discussed in [33] (see the fourth remark in Section 3.3 of that reference). Here, the truncated Fourier series is treated as a trigonometric polynomial in complex conjugate variables, and recursive formulas for polynomial compositions in the style of Knuth apply directly. As mentioned in [33], the truncation error introduced by stopping at a finite order can be rigorously bound via a posteriori analysis and the method can be applied for computer-assisted proofs employing Fourier methods. These ideas are used in [33] in order to study invariant KAM circles in the standard map.

The paper just mentioned appears in the book [34], which is based on the proceedings of an IMA conference held at the University of Cincinnati in April of 1986. Chapter 13 of the same book contains a discussion of software tools for analysis in function spaces. Such software packages need to be able to compute compositions with elementary functions as discussed above. Development of such software continues through the present and again a thorough survey of this literature is beyond the scope of the present work. More complete discussions of modern software libraries for computer-assisted proofs in Banach spaces can be found for example in [5, 35].

In the present study we apply the ideas of $\mathrm{AD}$ in a different way. Before we begin any numerical work, we introduce new coordinates by appending the differential equations for the composition term to the given system of differential equations. This results in an expanded system of polynomial differential equations to which we apply directly the methods of [1]. Our approach is then similar to that of [23], but adapted for computer-assisted proofs involving Fourier series. 
Techniques based on interpolation (rather than automatic differentiation) are also useful for evaluating compositions of Fourier series with transcendental functions, and such methods have been implemented in computer-assisted proofs. In particular we direct the reader to the work of $[36,37]$. These authors expand their transcendental nonlinearities as Chebeyshev polynomials, evaluate the polynomial compositions using discrete Fourier convolutions, and manage the truncation errors via classical interpolation error estimates. The present work is closely related to the work of [37], as there the authors also study periodic orbits of a celestial mechanics problem via Fourier series methods. Our AD treatment of nonlinearities is however different.

We remark that our automatic differentiation strategy for computing compositions will fail if the vector field were given by general rather than elementary functions (i.e. if we knew only the Fourier or Taylor coefficients of the vector field). This is because our method exploits the fact that the elementary functions are solutions of known differential equations. In more general settings one would have to employ an interpolation scheme as in [36,37]. General compositions are typical in renormalization theory, see for example the work of [38, 39, 40, 41, 42].

\subsection{Computer-Assisted Proof in Function Space and in Phase Space}

The literature on computer-assisted proof as a tool in nonlinear analysis is substantial and growing rapidly. A thorough review would take us far afield, and instead we direct the reader to several expository articles $[43,5,44,45]$ and refer also to the book by Tucker [25] for more scholarly discussion. In what follows we offer only a few clarifying remarks.

Techniques for a-posteriori validation of periodic motions can (roughly speaking) be put into one of two broad classifications: topological and functional analytic. A critical component of many topological methods is rigorous numerical integration. Rigorous integrators played a key role in the computer-assisted proof of chaotic motions for the Lorenz equations [46, 47, 48], and also in the computer-assisted solution of Smale's 14-th problem [49, 50]. The interested reader will want to consult the works of $[29,51,26]$ for general rigorous numerical integrators for ordinary differential equations and see also $[52,53,54]$ for algorithms which integrate parabolic partial differential equations. See also the discussion of the literature in the references just mentioned.

Other topological methods of computer-assisted proof apply degree/index arguments directly to the vector field (integration free methods). Tools based on the Wazewski retract principle [55], the Conley Index Theory [56], the local stable/unstable manifold arguments of MeGehee [57], and more recent generalizations to normally hyperbolic invariant manifolds [58] facilitate integration free computer-assisted analysis of equilibria, periodic orbits, local stable/unstable manifolds, connecting orbits, and symbolic dynamics of ordinary and partial differential equations. We refer the reader to the works of $[59,60,61,62,63]$ and the references discussed therein.

Methods for studying periodic orbits of differential equations using functional analysis are already outlined in Section 1.1. Functional analytic methods of computer-assisted proof go back to work of Cesari on Galerkin projections for periodic solutions [64, 65], and are exemplified also in Lanford's proof of the Feigenbaum conjectures [38].

At present we stress that topological methods apply fixed point/index arguments in phase space and result in a representation of the periodic orbit as a point or set in phase space with validated error bounds, and functional analytic techniques apply fixed point/index arguments in function space and result in a representation of the periodic orbit as a point or set in Banach space with validated error bounds. Both the topological and the functional analytic approaches answer the same question: namely "does there exist a periodic orbit near the results of this numerical simulation?" It is only the representation of the validated object which differs. Yet depending on what one does next with the computer-assisted results, the form of the representation could be an important consideration. We briefly indicate several situations where the quantitative information provided by a function space argument could be of interest. The list is suggestive rather than exhaustive.

- Poles in the complex plane: suppose that $\gamma:[0, T] \rightarrow \mathbb{R}^{n}$ is a $T$-periodic solution of the differential equation

$$
\gamma^{\prime}=f(\gamma),
$$

with $f$ an analytic function, and let $\left\{a_{k}\right\}_{k \in \mathbb{Z}}$ with each $a_{k} \in \mathbb{R}^{n}$ be the Fourier coefficients of $\gamma$. If the coefficients $a_{k}$ decay with some geometric rate $\nu>1$ (i.e. of $\left|a_{k}\right| \leq C \nu^{k}$ ) then $\gamma$ is real analytic and can be extended to a real analytic function on the complex strip of radius $T \log \nu / 2 \pi$. The radius of this strip then provides a lower bound on 
the location of any poles of $\gamma$ in the complex plane. The poles of a periodic orbit determine for example the "stiffness" of the differential equation near $\gamma$. Of course analyzing the poles of an analytic function has myriad other classical applications.

- Bootstrap bounds on derivatives: explicit knowledge of the Fourier series of an analytic function allows us to study also the derivatives via Cauchy bounds. The ability to compute derivatives is useful for obtaining observables such as the energy or for studying the functions maxima and minima.

- Stability/Floquet analysis: The stability of a periodic orbit can be determined by studying periodic solutions of the eigenvalue problem

$$
\frac{d}{d t} \xi(t)+\lambda \xi(t)-D f[\gamma(t)] \xi(t)=0,
$$

and it is noteworthy that this equation can again be studied using the techniques discussed in the present work, see for example $[66,16]$

Note that in order to study this problem using Fourier analysis it is necessary to know $\gamma$ exactly (in the sense of computer-assisted analysis "exactly" might mean that $\gamma$ is given by a known trigonometric polynomial plus an explicit error bound in some Banach space). Stability of periodic orbits can also be studied using rigorous integrators and cone conditions applied in a Poincare section. However the functional analytic approach yields a parameterization of the stable/unstable bundle, and one obtains analytic properties of the parameterized bundle in addition to the parameterized periodic orbit.

- Computer-assisted proof for periodic orbits in problem with no globally defined flow: the functional analytic approach to computer-assisted proof if based on solving equations rather than propagating initial conditions. Hence this approach sometimes applies in situations where there is no locally defined flow. For example computer-assisted proofs based on functional analytic techniques have been used to study periodic orbits for delay equations and ill-posed PDEs $[13,67,68]$. The automatic differentiation scheme proposed here could be modified to apply to some of these problems.

- Validated Fourier-Taylor computation of stable/unstable manifolds for periodic orbits: Suppose that $\xi:[0, T] \rightarrow \mathbb{R}^{n}$ and $\lambda \in \mathbb{R}$ solve the eigenvalue problem above. Then briefly, a covering map (or parameterization) $P:[0, T] \times$ $[-R, R] \rightarrow \mathbb{R}^{n}$ of the stable/unstable manifold for $\gamma$ satisfies the equation

$$
\frac{\partial}{\partial t} P(t, \sigma)+\lambda \sigma \frac{\partial}{\partial \sigma} P(t, \sigma)=f[P(t, \sigma)],
$$

subject to the constraints

$$
P(t, 0)=\gamma(t), \quad \text { and } \quad \frac{\partial}{\partial \sigma} P(t, \sigma)=\xi(t) .
$$

Solving this equation leads to an analytic Fourier-Taylor expansion of the stable/unstable manifold. These developments are discussed further in $[69,70,71]$. The results of the present work could be taken as the inputs for rigorous computations of stable/unstable manifolds of periodic orbits based on the Parameterization Method in non-polynomial systems. This will make the topic of a future study. We remark also that validated/Fourier-Taylor expansions of stable unstable manifolds of periodic orbits could be used to obtain or improve computer-assisted proofs involving applications of scattering maps [72] and Melnikov integrals [73].

A number of authors have developed computer assisted methods of proof based on KAM theory. See for example $[33,74,75]$, and the references therein. In particular these authors compute validated expansions of quasi periodic orbits/invariant tori and their stable/unstable manifolds. More complete discussion of this literature is found in the book of Haro, Candella, Figueras, Luque, and Mondelo [76], and also the book of Celletti [77]. KAM arguments for invariant circles, tori, and their whiskers often exploit computer-assisted Fourier analysis, and the automatic differentiation scheme proposed in the present work could be useful in this setting as well. 
To summarize: the functional analytic approach relies on the regularity of the object under study and is especially useful when, in addition to existence and localization results, we are interested also in either some quantitative analytic properties of the solution or in using the solution of one problem as input to another functional analytic argument. Especially if (as in the Parameterization Method) we desire a parametric representation of a dynamical object. On the other hand, topological methods are able to resolve objects with no manifold structure (strange attractors/invariant sets with Cantor-like topology) which have no smooth parametric representation. See especially [50, 46, 78, 79, 80]. For many problems in nonlinear analysis both topological and functional analytic methods of computer assisted proof are available, and practitioners will decide what tools to apply based a any number of considerations.

Remark 1. Periodic motions and computer-assisted proof in Celestial Mechanics: The N-body problem occupies a central place in mathematical physics going back to the time of Newton. Yet the problem is highly nonlinear and many interesting applications are non-perturbative. It is partly for these reasons that applications in celestial mechanics, and in particular applications involving the circular restricted three body problem, have received considerable attention from the computer-assisted proof community over the last decades. We refer the reader to the book of Celletti [77] for more scholarly discussion of the literature, especially with regards to quasi-periodic motions and KAM theory.

A number of authors have used computer-assisted nonlinear analysis to study periodic orbits for $N$-body problems. In addition to the work of [37] (already mentioned above) we mention the papers of [81,30,82], where families of Lyapunov orbits for the PCRTBP are studied with computer assistance. In addition to the existence of the periodic orbits the authors also prove the existence of topological horseshoes and symbolic dynamics between orbits. This work is extended in [83], and the author obtains the existence of transverse intersections between the stable and unstable manifolds of some Lyapunov orbits. The paper [84] develops a computer assisted proof of the existence and localization of a center manifold in the PCRTBP. The work of [85] studied the existence and stability of continuous branches of orbits for the CRTBP. Instabilities in the CRTBP have been studied with computer assistance in [86].

There are also computer-assisted results for periodic orbits in more general celestial mechanics problems. In [87] the authors study transition tori in an elliptic perturbation of the CRTBP. The series of papers $[88,89,90]$ provide computerassisted proofs of a number of choreography orbits in the full $N$-body problem. This discussion is by no means complete, and the interested reader can consult also the reference in the papers just mentioned.

\subsection{Example of Automatic Differentiation for Taylor Series}

We recall the basic notion of automatic differentiation for Taylor series. Consider the formal power series

$$
f(z)=\sum_{n=0}^{\infty} a_{n}\left(z-z_{0}\right)^{n}, \quad \text { and } \quad g(z)=\sum_{n=0}^{\infty} b_{n}\left(z-z_{0}\right)^{n} .
$$

We denote the $n$-th power series coefficient by $(f)_{n}=a_{n}$. With $\alpha \in \mathbb{C}$, the standard arithmetic operations extend to power series "term by term" as

$$
\begin{aligned}
(f+g)_{n} & =a_{n}+b_{n} \\
(\alpha f)_{n} & =\alpha a_{n} \\
\left(f^{\prime}\right)_{n} & =(n+1) a_{n+1} \\
(f \cdot g)_{n} & =\sum_{k=0}^{n} a_{n-k} b_{k}
\end{aligned}
$$

and we have that

$$
f=g \quad \text { if and only if } \quad a_{n}=b_{n} \text { for all } n \geq 0 .
$$

We now wish to extend these basic notions to include the elementary functions. For example, let $e^{z}$ denote the exponential function and consider the problem of computing $\left(e^{f}\right)_{n}$. A natural idea would be to expand $e^{z}$ using its Taylor 
series, so that the composition with $f$ is computed by repeated application of Equation (1.6) in order to obtain the coefficient of $f^{k}$.

This turns out to be an inefficient way of obtaining the composition, and if we exploit further properties of the exponential function, we arrive to a more effective scheme. Note that $\left(e^{f}\right)_{0}=e^{f\left(z_{0}\right)}=e^{a_{0}}$. Introducing the new power series variable

$$
e^{f(z) \stackrel{\text { def }}{=}} g(z)=\sum_{n=0}^{\infty} b_{n}\left(z-z_{0}\right)^{n}
$$

observe that

$$
\frac{d}{d z} g(z)=e^{f(z)} f^{\prime}(z)=g(z) f^{\prime}(z),
$$

that is

$$
\sum_{n=0}^{\infty}(n+1) b_{n+1}\left(z-z_{0}\right)^{n}=\sum_{n=0}^{\infty}\left(\sum_{k=0}^{n} b_{n-k}(k+1) a_{k+1}\right)\left(z-z_{0}\right)^{n} .
$$

Matching like powers of $\left(z-z_{0}\right)$ yields

$$
\left(e^{f}\right)_{n}=\left\{\begin{array}{ccc}
e^{a_{0}} & \text { if } & n=0 \\
\frac{1}{n} \sum_{k=1}^{n} k a_{k}\left(e^{f}\right)_{n-k} & \text { if } & n \geq 1
\end{array}\right.
$$

Note that we obtain the coefficients of $e^{f}$ at the cost (in arithmetic operations) of multiplying two power series. The example of $\left(f^{\alpha}\right)_{n}$ is worked out in detail in [20] using similar arguments. One can work out the power series coefficients of the composition with any elementary function. For more examples see [23], especially the discussion of Proposition 2.1 in that work.

\subsection{An Automatic Differentiation Scheme for Fourier Series}

Now suppose that $f(z), g(z)$ are given by the Fourier series

$$
f(z)=\sum_{n \in \mathbb{Z}} a_{n} e^{i n z}, \quad \text { and } \quad g(z)=\sum_{n \in \mathbb{Z}} b_{n} e^{i n z} .
$$

Then, formally speaking, we have the term-by-term relations

$$
\begin{aligned}
(f+g)_{n} & =a_{n}+b_{n} \\
(\alpha f)_{n} & =\alpha a_{n} \\
\left(f^{\prime}\right)_{n} & =i n a_{n} \\
(f \cdot g)_{n} & =\sum_{k \in \mathbb{Z}} a_{n-k} b_{k}
\end{aligned}
$$

and that

$$
f=g \quad \text { if and only if } \quad a_{n}=b_{n} \text { for all } n \in \mathbb{Z} .
$$

In analogy with the case of power series suppose that we desire

$$
g(z) \stackrel{\text { def }}{=} e^{f(z)}=\sum_{n \in \mathbb{Z}} b_{n} e^{i n z} .
$$

Returning to Equation (1.8) and in this case applying Equations (1.11), (1.12) and (1.13) we see that the desired Fourier coefficients are related by the equations

$$
i n b_{n}=\sum_{k \in \mathbb{Z}} i k b_{n-k} a_{k}, \quad n \in \mathbb{Z},
$$


subject to the initial condition

$$
g(0)=e^{f(0)}
$$

that is

$$
\sum_{n \in \mathbb{Z}} b_{n}=e^{\sum_{n \in \mathbb{Z}} a_{n}} .
$$

We treat Equations (1.14), (1.15) as a system of infinitely many coupled nonlinear equations in infinitely many unknowns $b_{n}$. Truncating the system to finite order $N$, we can apply a Newton scheme in order to numerically approximate the desired Fourier coefficients $b_{n}$ for $-N \leq n \leq N$. This discussion extends to other elementary functions by appending appropriate differential equations. Other spectral methods (for example Chebyshev series) can be treated by the same method.

\section{Planar Circular Restricted Three-Body Problem}

A classical problem in celestial mechanics is the Planar Circular Restricted Three-Body Problem (PCRTBP). We provide a brief description of the problem and refer the reader to the book by Meyer and Hall [91] for a more complete treatment and discussion of the literature. In this problem, one considers two bodies of mass $m_{1}>m_{2}>0$ moving in a circular Keplerian orbit. These are called the primary and secondary bodies respectively. Let $\mu=m_{1} /\left(m_{1}+m_{2}\right)$ denote the mass ratio and choose coordinates so that the circular orbit lies in the $X, Y$ plane with center of mass at the origin. Since orbits are circular, the line determined by the masses passes through the center of mass (that is the origin) and rotates at a constant frequency. One can define a rotating coordinate system with the $x$ axis determined by the line between the two masses. The coordinates are rescaled so that distances between the massive bodies and the origin are $\mu$ and $1-\mu$ respectively. Hence the bodies are positioned at $-\mu$ and $1-\mu$ along the fixed rotating line $x$. This situation is illustrated in Figures 1 and 2 .

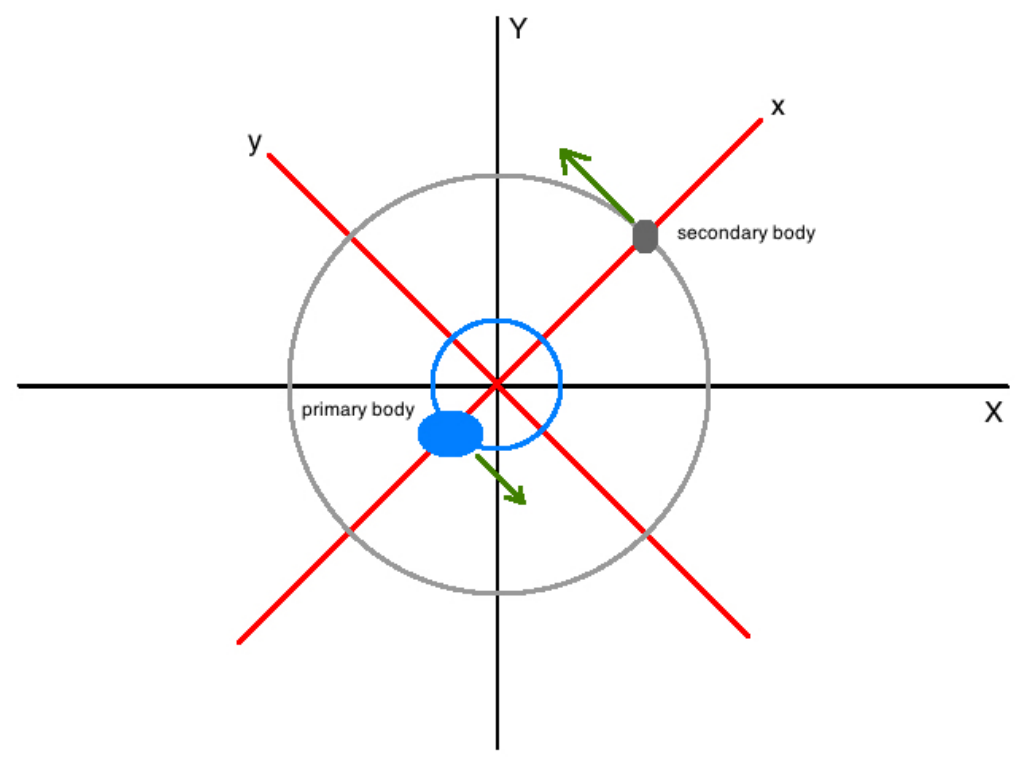

Figure 1: A massive primary body and a smaller secondary body in circular motion. The $X, Y$ coordinates (black axes) illustrate the standard Galilean center of mass coordinate frame. The $x, y$ coordinates (red axes) illustrate a co-rotating frame. In the rotating frame, the primary and secondary bodies lie always on the $x$-axis.

The massive bodies are now referred to collectively as "the primaries". A third and massless particle (sometimes called a "test particle") is placed in the plane of motion of the primaries. The massless particle moves in the resulting gravitational 


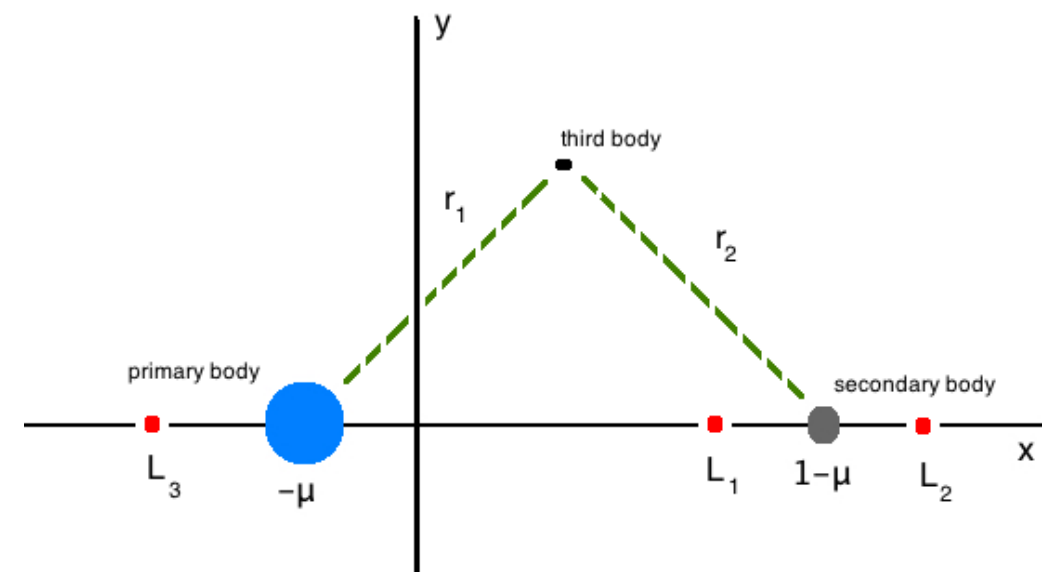

Figure 2: In the co-rotating (non-inertial) frame, we introduce a third and massless particle. The magnitude of the distance from the massless particle to the primary body is $r_{1}$ and the magnitude of the distance from the massless particle to the secondary particle is $r_{2}$. The massless particle is influenced by the gravitational fields of the primary and secondary bodies, however the massless body does not affect the orbits of the massive bodies. In the rotating reference frame, there are three collinear equilibrium points on the $x$-axis which are denoted $L_{1}, L_{2}, L_{3}$.

field without disturbing the Keplerian motion of the primaries, that is without creating any gravitational field of its own. Writing Newton's laws for the motion of the massless particle (transformed to the non-inertial co-rotating reference frame) gives the system of two second order ordinary differential equations

$$
\left\{\begin{array}{l}
x^{\prime \prime}=2 y^{\prime}+\frac{\partial V}{\partial x} \\
y^{\prime \prime}=-2 x^{\prime}+\frac{\partial V}{\partial y},
\end{array}\right.
$$

where

$$
V(x, y) \stackrel{\text { def }}{=} \frac{1}{2}\left(x^{2}+y^{2}\right)+\frac{1-\mu}{r_{1}(x, y)}+\frac{\mu}{r_{2}(x, y)}
$$

and

$$
\left\{\begin{array}{l}
r_{1}(x, y) \stackrel{\text { def }}{=} \sqrt{(x+\mu)^{2}+y^{2}} \\
r_{2}(x, y) \stackrel{\text { def }}{=} \sqrt{(x-1+\mu)^{2}+y^{2}}
\end{array}\right.
$$

Letting $x_{1} \stackrel{\text { def }}{=} x, x_{2} \stackrel{\text { def }}{=} x^{\prime}, x_{3} \stackrel{\text { def }}{=} y$ and $x_{4} \stackrel{\text { def }}{=} y^{\prime}$, the system $(2.1)$ becomes

$$
\left\{\begin{array}{l}
x_{1}^{\prime}=x_{2} \\
x_{2}^{\prime}=2 x_{4}+\frac{\partial V}{\partial x} \\
x_{3}^{\prime}=x_{4} \\
x_{4}^{\prime}=-2 x_{2}+\frac{\partial V}{\partial y} .
\end{array}\right.
$$

The system has at most five equilibrium solutions, three of which lie on the $x$-axis. The three equilibrium points on the $x$-axis are called the collinear equilibria and are denoted $L_{1}, L_{2}, L_{3}$. The collinear equilibria have saddle-center stability. The center manifold of each collinear equilibrium point is foliated by a family of hyperbolic periodic orbits referred to as the Lyapunov orbits. The existence of a one parameter family of Lyapunov orbits in a small neighborhood of each of the colinear equilibrium is classical and can be proved by a center manifold argument, see for example [92]. The Lyapunov orbits are parametrized by energy/frequency, are the main point of study in the remainder of the present work. Our main results concern the existence of Lyapunov orbits far from the perturbative regime. 


\subsection{Equivalent Polynomial Vector Field Formulation and the Automatic Differentiation of Fourier Series for the CRTBP}

Next, we want to transform (2.2) into a polynomial system. In order to do so, we first take the partial derivatives of $V(x, y)$, that is

$$
\left\{\begin{array}{l}
\frac{\partial V}{\partial x}=x-\frac{(1-\mu)(x+\mu)}{\left((x+\mu)^{2}+y^{2}\right)^{\frac{3}{2}}}-\frac{\mu(x-1+\mu)}{\left((x-1+\mu)^{2}+y^{2}\right)^{\frac{3}{2}}} \\
\frac{\partial V}{\partial y}=y-\frac{(1-\mu) y}{\left((x+\mu)^{2}+y^{2}\right)^{\frac{3}{2}}}-\frac{\mu y}{\left((x-1+\mu)^{2}+y^{2}\right)^{\frac{3}{2}}} .
\end{array}\right.
$$

We then let $x_{5} \stackrel{\text { def }}{=} \frac{1}{\sqrt{\left(x_{1}+\mu\right)^{2}+x_{3}^{2}}}$ and $x_{6} \stackrel{\text { def }}{=} \frac{1}{\sqrt{\left(x_{1}-1+\mu\right)^{2}+x_{3}^{2}}}$. By differentiating these with respect to $t$, we get

$$
x_{5}^{\prime}(t)=-\frac{(x+\mu) x^{\prime}+y y^{\prime}}{\left((x+\mu)^{2}+y^{2}\right)^{\frac{3}{2}}}=-x_{5}^{3}\left(\left(x_{1}+\mu\right) x_{2}+x_{3} x_{4}\right)
$$

and

$$
x_{6}^{\prime}(t)=-\frac{(x-1+\mu) x^{\prime}+y y^{\prime}}{\left((x-1+\mu)^{2}+y^{2}\right)^{\frac{3}{2}}}=-x_{6}^{3}\left(\left(x_{1}-1+\mu\right) x_{2}+x_{3} x_{4}\right) .
$$

Plugging $x_{5}$ and $x_{6}$ into (2.2) and adding our expressions for $x_{5}^{\prime}$ and $x_{6}^{\prime}$ into the mix, we get our final quintic polynomial vector field

$$
\left\{\begin{array}{l}
x_{1}^{\prime}=x_{2} \\
x_{2}^{\prime}=2 x_{4}+x_{1}-(1-\mu)\left(x_{1}+\mu\right) x_{5}^{3}-\mu x_{6}^{3}\left(x_{1}-1+\mu\right) \\
x_{3}^{\prime}=x_{4} \\
x_{4}^{\prime}=-2 x_{2}+x_{3}-(1-\mu) x_{3} x_{5}^{3}-\mu x_{3} x_{6}^{3} \\
x_{5}^{\prime}=-x_{5}^{3}\left(\left(x_{1}+\mu\right) x_{2}+x_{3} x_{4}\right) \\
x_{6}^{\prime}=-x_{6}^{3}\left(\left(x_{1}-1+\mu\right) x_{2}+x_{3} x_{4}\right)
\end{array}\right.
$$

where the extra conditions

$$
\begin{aligned}
& x_{5}(0)=\frac{1}{\sqrt{\left(x_{1}(0)+\mu\right)^{2}+x_{3}(0)^{2}}} \\
& x_{6}(0)=\frac{1}{\sqrt{\left(x_{1}(0)-1+\mu\right)^{2}+x_{3}(0)^{2}}}
\end{aligned}
$$

need to be imposed.

Let us now introduce the operator (1.1) whose solutions correspond to periodic solutions of (2.1). Once this operator is introduced, we use the theory of Section 3 of [1] to compute rigorously its solutions.

\subsection{The Operator Equation $F(a)=0$}

Given a fixed period $2 \pi / \omega$, where $\omega$ is the frequency, we look for periodic orbits of $(2.1)$ satisfying $x^{\prime}(0)=y(0)=0$. Exploiting the well known symmetry of Equation (2.2) allowse us to take $x_{1}, x_{4}, x_{5}, x_{6}$ as cosine series and $x_{2}, x_{3}$ are sine series, that is

$$
x_{j}(t)=\sum_{k \in \mathbb{Z}}\left(a_{j}\right)_{k} e^{i k \omega t}=\left(a_{j}\right)_{0}+2 \sum_{k=1}^{\infty}\left(a_{j}\right)_{k} \cos (k \omega t), \quad \text { for } j=1,4,5,6
$$

with $\left(a_{j}\right)_{-k} \stackrel{\text { def }}{=}\left(a_{j}\right)_{k} \in \mathbb{R}$ for $j=1,4,5,6$, and

$$
x_{j}(t)=\sum_{k \in \mathbb{Z}} i\left(a_{j}\right)_{k} e^{i k \omega t}=-2 \sum_{k=1}^{\infty}\left(a_{j}\right)_{k} \sin (k \omega t), \quad \text { for } j=2,3,
$$

with $\left(a_{j}\right)_{-k} \stackrel{\text { def }}{=}-\left(a_{j}\right)_{k} \in \mathbb{Z}$ for $j=2,3$. While the cosine and sine series only have positive indices, it is important to also consider negative indices, so that we can calculate discrete convolutions the usual way. For instance, 


$$
x_{1}(t) x_{2}(t) x_{5}^{3}(t)=\sum_{k \in \mathbb{Z}}\left(a_{1}\left(i a_{2}\right) a_{5}^{3}\right)_{k} e^{i k \omega t}=-2 \sum_{k=1}^{\infty}\left(a_{1} a_{2} a_{5}^{3}\right)_{k} \sin (k \omega t),
$$

where

$$
\left(a_{1} a_{2} a_{5}^{3}\right)_{k}=\sum_{\substack{k_{1}+k_{2}+k_{3}+k_{4}+k_{5}=k \\ k_{i} \in \mathbb{Z}}}\left(a_{1}\right)_{k_{1}}\left(a_{2}\right)_{k_{2}}\left(a_{5}\right)_{k_{3}}\left(a_{5}\right)_{k_{4}}\left(a_{5}\right)_{k_{5}}
$$

and where $\left(a_{1} a_{2} a_{5}^{3}\right)_{-k}=-\left(a_{1} a_{2} a_{5}^{3}\right)_{k}$ follows from the fact that $\left(a_{1}\right)_{-k}=\left(a_{1}\right)_{k},\left(a_{2}\right)_{-k}=-\left(a_{2}\right)_{k}$ and $\left(a_{5}\right)_{-k}=\left(a_{5}\right)_{k}$.

Now that we have a Fourier representation of our solutions, all we need to do is find the coefficients. To do this, we first substitute our series into (2.3). Since the equations must be verified for all values of $t$, we have that our vector function solves (2.3) if and only if the coefficients correspond on both sides of the equation. So, by putting everything on the same side of the equation, we must solve $\left(F_{j}\right)_{k}=0$ for $j=1, \ldots, 6$, where for $k \geq 1$,

$$
\left\{\begin{aligned}
\left(F_{1}\right)_{k} & \stackrel{\text { def }}{=} k \omega\left(a_{1}\right)_{k}-\left(a_{2}\right)_{k} \\
\left(F_{2}\right)_{k} & \stackrel{\text { def }}{=} k \omega\left(a_{2}\right)_{k}+2\left(a_{4}\right)_{k}+\left(a_{1}\right)-(1-\mu)\left(a_{1} a_{5}^{3}\right)_{k}-\left(\mu-\mu^{2}\right)\left(a_{5}^{3}\right)_{k} \\
& -\mu\left(a_{1} a_{6}^{3}\right)_{k}+\left(\mu-\mu^{2}\right)\left(a_{6}^{3}\right)_{k} \\
\left(F_{3}\right)_{k} & \stackrel{\text { def }}{=} k \omega\left(a_{3}\right)_{k}+\left(a_{4}\right)_{k} \\
\left(F_{4}\right)_{k} & \stackrel{\text { def }}{=} k \omega\left(a_{4}\right)_{k}+2\left(a_{2}\right)_{k}-\left(a_{3}\right)_{k}+(1-\mu)\left(a_{3} a_{5}^{3}\right)_{k}+\mu\left(a_{3} a_{6}^{3}\right)_{k} \\
\left(F_{5}\right)_{k} & \stackrel{\text { def }}{=} k \omega\left(a_{5}\right)_{k}+\left(a_{1} a_{2} a_{5}^{3}\right)_{k}+\left(a_{3} a_{4} a_{5}^{3}\right)_{k}+\mu\left(a_{2} a_{5}^{3}\right)_{k} \\
\left(F_{6}\right)_{k} & \stackrel{\text { def }}{=} k \omega\left(a_{6}\right)_{k}+\left(a_{1} a_{2} a_{6}^{3}\right)_{k}+\left(a_{3} a_{4} a_{6}^{3}\right)_{k}+(\mu-1)\left(a_{2} a_{6}^{3}\right)_{k} .
\end{aligned}\right.
$$

Since $x_{2}, x_{3} \in \mathbb{R}$ are expressed as sine series, their constant Fourier coefficients, given respectively by $\left(a_{2}\right)_{0}$ and $\left(a_{3}\right)_{0}$, are zero. However, to simplify the setup, we consider $\left(a_{2}\right)_{0}$ and $\left(a_{3}\right)_{0}$ as variables and impose them to be zero within the operator. Therefore, for $k=0$, we set

$$
\left\{\begin{aligned}
&\left(F_{1}\right)_{0} \stackrel{\text { def }}{=}\left(a_{2}\right)_{0} \\
&\left(F_{2}\right)_{0} \stackrel{\text { def }}{=} \\
& 2\left(a_{4}\right)_{0}+\left(a_{1}\right)-(1-\mu)\left(a_{1} a_{5}^{3}\right)_{0}-\left(\mu-\mu^{2}\right)\left(a_{5}^{3}\right)_{0} \\
&-\mu\left(a_{1} a_{6}^{3}\right)_{0}+\left(\mu-\mu^{2}\right)\left(a_{5}^{3}\right)_{0} \\
&\left(F_{3}\right)_{0} \stackrel{\text { def }}{=}\left(a_{4}\right)_{0} \\
&\left(F_{4}\right)_{0} \stackrel{\text { def }}{=}\left(a_{3}\right)_{0} \\
&\left(F_{5}\right)_{0} \stackrel{\text { def }}{=} \eta_{1} \\
&\left(F_{6}\right)_{0} \stackrel{\text { def }}{=} \eta_{2},
\end{aligned}\right.
$$

where $\eta_{1}$ and $\eta_{2}$ depends on the value of $x_{1}(0)+\mu$. More explicitly,

$$
\begin{gathered}
\eta_{1} \stackrel{\text { def }}{=}\left\{\begin{array}{cl}
x_{5}(0)\left(x_{1}(0)+\mu\right)-1, & \text { if } x_{1}(0)+\mu>1 \\
x_{5}(0)\left(x_{1}(0)+\mu\right)-1, & \text { if } x_{1}(0)+\mu \in(0,1) \\
-x_{5}(0)\left(x_{1}(0)+\mu\right)-1, & \text { if } x_{1}(0)+\mu<0
\end{array}\right. \\
\eta_{2} \stackrel{\text { def }}{=}\left\{\begin{array}{cc}
x_{6}(0)\left(x_{1}(0)+\mu-1\right)-1, & \text { if } x_{1}(0)+\mu>1 \\
-x_{6}(0)\left(x_{1}(0)+\mu-1\right)-1, & \text { if } x_{1}(0)+\mu \in(0,1) \\
-x_{6}(0)\left(x_{1}(0)+\mu-1\right)-1, & \text { if } x_{1}(0)+\mu<0,
\end{array}\right.
\end{gathered}
$$

where $x_{j}(0)=\left(a_{j}\right)_{0}+2 \sum_{k>1}\left(a_{j}\right)_{k}$ for $j=1,5,6$. In all cases, $\eta_{1}=0$ ensures that (2.4) holds while $\eta_{2}=0$ ensures that $(2.5)$ holds. Note also that $\left(F_{4}\right)_{0}=0$ forces $x_{3}(0)$ to vanish.

For $j=1, \ldots, 6$, let $a_{j} \stackrel{\text { def }}{=}\left(\left(a_{j}\right)_{k}\right)_{k \geq 0}$ and $F_{j} \stackrel{\text { def }}{=}\left(\left(F_{j}\right)_{k}\right)_{k \geq 0}$. Moreover, set

$$
a \stackrel{\text { def }}{=}\left(a_{1}, a_{2}, a_{3}, a_{4}, a_{5}, a_{6}\right) \text { and } F \stackrel{\text { def }}{=}\left(F_{1}, F_{2}, F_{3}, F_{4}, F_{5}, F_{6}\right) .
$$

From the above discussions, computing periodic solutions of the planar circular restricted 3-body problem is equivalent to finding solutions of

$$
F(a)=0,
$$

where the coefficients of $F(a)$ are given by (2.7) and (2.6).

Let us now introduce the Banach space $X$ on which we look for solutions of (2.10). 


\subsection{The Banach Space $X$}

Since (2.3) is a real analytic vector field, any periodic solution is real analytic. Therefore, the Fourier coefficients of the components of any periodic solution decay to zero exponentially fast. This fundamental fact justifies the choice of Banach space on which we solve (2.10). Given an exponential decay rate $\nu \geq 1$, let

$$
\ell_{\nu}^{1} \stackrel{\text { def }}{=}\left\{c=\left(c_{k}\right)_{k \geq 0} \mid c_{k} \in \mathbb{R} \text { and }\|c\|_{\nu} \stackrel{\text { def }}{=} \sum_{k \geq 0}\left|c_{k}\right| \nu^{k}<\infty\right\} .
$$

A consequence of the definition of the above space is that a sequence $c \in \ell_{\nu}^{1}$ must have that its coefficients decay (at infinity) to zero faster than the geometric decay rate $\nu^{-k}$. Define the Banach space

$$
X \stackrel{\text { def }}{=}\left(\ell_{\nu}^{1}\right)^{6}=\left\{a=\left(a_{1}, a_{2}, a_{3}, a_{4}, a_{5}, a_{6}\right) \mid a_{j} \in \ell_{\nu}^{1}, \quad j=1, \ldots, 6\right\}
$$

endowed with the norm

$$
\|a\|_{X}=\max _{j=1, \ldots, 6}\left\{\left\|a_{j}\right\|_{\nu}\right\} .
$$

To derive the bounds necessary to prove existence of solutions of (2.10) within the Banach space $X$ defined by (2.11), we will use the following fundamental result.

Lemma 2. Let $\nu \geq 1$ and $a_{1}, a_{2} \in \ell_{\nu}^{1}$. Consider any bi-infinite sequences $\tilde{a}_{1}=\left(\left(\tilde{a}_{1}\right)_{k}\right)_{k \in \mathbb{Z}}, \tilde{a}_{2}=\left(\left(\tilde{a}_{2}\right)_{k}\right)_{k \in \mathbb{Z}}$ with indices so that $\left(\tilde{a}_{j}\right)_{k}=\left(a_{j}\right)_{k}$ and $\left|\left(\tilde{a}_{j}\right)_{-k}\right|=\left|\left(a_{j}\right)_{k}\right|$ for $j=1,2$ and for all $k \geq 0$. Then $\left(\left(\tilde{a}_{1} * \tilde{a}_{2}\right)_{k}\right)_{k \geq 0} \in \ell_{\nu}^{1}$ and

$$
\left\|\left(\left(\tilde{a}_{1} * \tilde{a}_{2}\right)_{k}\right)_{k \geq 0}\right\|_{\nu} \leq 4\left\|a_{1}\right\|_{\nu}\left\|a_{2}\right\|_{\nu} .
$$

Proof.

$$
\begin{aligned}
\left\|\left(\left(\tilde{a}_{1} * \tilde{a}_{2}\right)_{k}\right)_{k \geq 0}\right\|_{\nu} & =\sum_{k \geq 0}\left|\left(\tilde{a}_{1} * \tilde{a}_{2}\right)_{k}\right| \nu^{k}=\sum_{k \geq 0}\left|\sum_{\substack{k_{1}+k_{2}=k \\
k_{1}, k_{2} \in \mathbb{Z}}}\left(\tilde{a}_{1}\right)_{k_{1}}\left(\tilde{a}_{2}\right)_{k_{2}}\right| \nu^{k} \\
& \leq \sum_{k \geq 0} \sum_{\substack{k_{1}+k_{2}=k \\
k_{1}, k_{2} \in \mathbb{Z}}}\left|\left(\tilde{a}_{1}\right)_{k_{1}}\right|\left|\left(\tilde{a}_{2}\right)_{k_{2}}\right| \nu^{k} \leq 4 \sum_{k \geq 0} \sum_{\substack{k_{1}+k_{2}=k \\
k_{1}, k_{2} \geq 0}}\left|\left(a_{1}\right)_{k_{1}}\right| \nu^{k_{1}}\left|\left(a_{2}\right)_{k_{2}}\right| \nu^{k_{2}} \\
& \leq 4\left(\sum_{k_{1} \geq 0}\left|\left(a_{1}\right)_{k_{1}}\right| \nu^{k_{1}}\right)\left(\sum_{k_{2} \geq 0}\left|\left(a_{2}\right)_{k_{2}}\right| \nu^{k_{2}}\right)=4\left\|a_{1}\right\|_{\nu}\left\|a_{2}\right\|_{\nu} .
\end{aligned}
$$

Remark 2. The bound (2.12) holds for discrete convolutions involving any combination of cosine sequences (that is sequences extended over negative indices using the rule $b_{-k}=b_{k}$ ) and sine sequences (extended over negative indices using the rule $b_{-k}=-b_{k}$ ).

Instead of solving (2.10) directly, we introduce a fixed point equation of the form $T(a)=a-A F(a)$ (where $A$ is an injective linear operator to be defined) whose fixed points are in one-to-one correspondence with the zeroes of $F$.

\subsection{Definition of the Approximate Inverse Operator $A$}

Assume that using a finite-dimensional projection $F^{(m)}: \mathbb{R}^{6 m} \rightarrow \mathbb{R}^{6 m}$ of $(2.10)$, we applied Newton's method to find a numerical solution $\bar{a}=\left(\bar{a}_{1}, \ldots, \bar{a}_{6}\right) \in \mathbb{R}^{6 m}$ such that $F^{(m)}(\bar{a}) \approx 0$. From now on, we abuse slightly the notation by identifying the point $\bar{a}_{j}=\left(\left(\bar{a}_{j}\right)_{-m+1}, \ldots,\left(\bar{a}_{j}\right)_{m-1}\right) \in \mathbb{R}^{m}$ with the point $\bar{a}_{j} \in \ell_{\nu}^{1}$ by padding by zeros, that is

$$
\bar{a}_{j}=\left(\cdots, 0, \cdots, 0,\left(\bar{a}_{j}\right)_{-m+1}, \ldots,\left(\bar{a}_{j}\right)_{m-1}, 0, \cdots, 0, \cdots\right) \in \ell_{\nu}^{1} .
$$


Denote $D F(\bar{a})=\left\{D_{a_{i}} F_{j}(\bar{a})\right\}_{i, j=1}^{6}$, where each component of $D F(\bar{a})$ is a linear operator such that $D_{a_{i}} F_{j}(\bar{a}): \ell_{\nu}^{1} \rightarrow \ell_{\nu^{\prime}}^{1}$ are linear operators with $\nu^{\prime}<\nu$. An intermediate first step is to consider $A^{\dagger}$ an approximation of $D F(\bar{a})$. The motivation for doing this is twofold. First, the derivative $D F(\bar{a})$ has many off diagonal terms that are small compared to the diagonal terms, and hence there are eventually diagonal dominant operators that approximate it well. Second, considering $A^{\dagger}$ will simplify the computing bounds for $D T(\bar{a}+b) c$ which we will decompose as $D T(\bar{a}+b) c=(I-A D F(\bar{a}+b)) c=$ $\left(I-A A^{\dagger}\right) c-A\left(D F(\bar{a}+b)-A^{\dagger}\right) c$. Indeed, each term of the sum will be bounded separately.

Hence, we first approximate $D F(\bar{a})$ with the operator $A^{\dagger}=\left\{A_{j, a_{i}}^{\dagger}\right\}_{i, j=1}^{6}$ which acts on $b=\left(b_{i}\right)_{i=1}^{6}$ component-wise as $\left(A^{\dagger} b\right)_{j}=\sum_{i=1}^{6} A_{j, a_{i}}^{\dagger} b_{i}$ for $j=1, \ldots, 6$, where $A_{j, a_{i}}^{\dagger} b_{i} \in \ell_{\nu^{\prime}}^{1}$ is defined component-wise by

$$
\left(A_{j, a_{i}}^{\dagger} b_{i}\right)_{k}=\left\{\begin{aligned}
\left(D_{a_{i}} F_{j}^{(m)}(\bar{a}) b_{i}^{(m)}\right)_{k}, & 0 \leq k<m \\
\delta_{i, j} \omega k\left(b_{i}\right)_{k}, & k \geq m .
\end{aligned}\right.
$$

Let $A^{(m)}$ be a finite-dimensional approximate inverse of $D F^{(m)}(\bar{a})$ which is obtained numerically. Define the decomposition $A^{(m)}=\left\{A_{j, a_{i}}^{(m)}\right\}_{i, j=1}^{6} \in \mathbb{R}^{6 m} \times \mathbb{R}^{6 m}$, where $A_{j, a_{i}}^{(m)} \in \mathbb{R}^{m} \times \mathbb{R}^{m}$. We define the approximate inverse $A$ of the infinite-dimensional operator $D F(\bar{a})$ by $A=\left\{A_{j, a_{i}}\right\}_{i, j=1}^{6}$, that is

$$
A=\left(\begin{array}{cccccc}
A_{1, a_{1}} & A_{1, a_{2}} & A_{1, a_{3}} & A_{1, a_{4}} & A_{1, a_{5}} & A_{1, a_{6}} \\
A_{2, a_{1}} & A_{2, a_{2}} & A_{2, a_{3}} & A_{2, a_{4}} & A_{2, a_{5}} & A_{2, a_{6}} \\
A_{3, a_{1}} & A_{3, a_{2}} & A_{3, a_{3}} & A_{3, a_{4}} & A_{3, a_{5}} & A_{3, a_{6}} \\
A_{4, a_{1}} & A_{4, a_{2}} & A_{4, a_{3}} & A_{4, a_{4}} & A_{4, a_{5}} & A_{4, a_{6}} \\
A_{5, a_{1}} & A_{5, a_{2}} & A_{5, a_{3}} & A_{5, a_{4}} & A_{5, a_{5}} & A_{5, a_{6}} \\
A_{6, a_{1}} & A_{6, a_{2}} & A_{6, a_{3}} & A_{6, a_{4}} & A_{6, a_{5}} & A_{6, a_{6}}
\end{array}\right) .
$$

The linear operator $A$ acts on $b=\left(b_{i}\right)_{i=1}^{6} \in X=\left(\ell_{\nu}^{1}\right)^{6}$ component-wise as $(A b)_{j}=\sum_{i=1}^{6} A_{j, a_{i}} b_{i} \in \ell_{\nu}^{1}$ for $j=1, \ldots, 6$ with $A_{j, a_{i}} b_{i} \in \ell_{\nu}^{1}$ defined component-wise by

$$
\left(A_{j, a_{i}} b_{i}\right)_{k}=\left\{\begin{aligned}
\left(A_{j, a_{i}}^{(m)} b_{i}^{(m)}\right)_{k}, & 0 \leq k<m \\
\frac{\delta_{i, j}}{\omega k}\left(b_{i}\right)_{k}, & k \geq m .
\end{aligned}\right.
$$

Having defined $A$ piece by piece, we can now define the Newton-like operator by

$$
T(a)=a-A F(a) .
$$

We show existence of fixed points of $T$ with the radii polynomial approach.

\subsection{The Radii Polynomial Approach for Periodic Orbits}

In this section, we essentially follow the approach introduced in Section 3 of [1]. This is why we omit many technical details.

Given $\bar{a}=\left(\bar{a}_{1}, \ldots, \bar{a}_{6}\right) \in X$, with $\bar{a}_{j}=\left(\cdots, 0, \cdots, 0,\left(\bar{a}_{j}\right)_{-m+1}, \ldots,\left(\bar{a}_{j}\right)_{m-1}, 0, \cdots, 0, \cdots\right) \in \ell_{\nu}^{1}$, define the bounds

$$
\begin{gathered}
Y=\left(Y_{1}, \ldots, Y_{6}\right) \in \mathbb{R}^{6} \\
Z(r)=\left(Z_{1}(r), \ldots, Z_{6}(r)\right) \in \mathbb{R}^{6}
\end{gathered}
$$

such that

$$
\left\|(T(\bar{a})-\bar{a})_{j}\right\|_{\nu} \leq Y_{j}, \quad \sup _{b, c \in B(r)}\left\|\left(D T_{j}(\bar{a}+b) c\right)\right\|_{\nu} \leq Z_{j}(r), \text { for } j=1, \ldots, 6 .
$$

The following result is proved in [1]. 
Proposition 3. Consider $Y, Z(r) \in \mathbb{R}^{6}$ satisfying (2.15). If there exists a radius $r>0$ such that $\max _{j=1, \ldots, 6}\left\{Z_{j}(r)+Y_{j}\right\}<r$, then $T: B_{\bar{a}}(r) \rightarrow B_{\bar{a}}(r)$ is a contraction mapping. Moreover, there exists a unique $\tilde{a} \in B_{\bar{a}}(r)$ such that $F(\tilde{a})=0$.

Definition 1. Given bounds $Y$ and $Z(r)$ satisfying $(2.15)$, define $p_{1}(r), \ldots, p_{6}(r)$ by

$$
p_{j}(r) \stackrel{\text { def }}{=} Z_{j}(r)-r+Y_{j} \text {. }
$$

If for each $j=1, \ldots, 6$, the bound $Z_{j}(r)$ is a polynomial in $r$, then $p_{j}(r)$ is a polynomial in $r$. In this case, the polynomials $p_{1}(r), \ldots, p_{6}$ are called the radii polynomials.

The definition of the radii polynomials is based on the assumption that each component of the bound $Z(r)$ can be obtained as a polynomial in $r$. We refer to Remark 1 in [1] for a justification of this assumption.

The next result, whose proof can be found in [1] provides an efficient mean of obtaining sets on which the Newton-like operator (2.14) is a contraction mapping.

Proposition 4. For a given exponential decay rate $\nu \geq 1$, construct the radii polynomials $p_{j}=p_{j}(r)$ for $j=1, \ldots, 6$ of Definition 1. Define

$$
\mathcal{I} \stackrel{\text { def }}{=} \bigcap_{j=1}^{6}\left\{r>0 \mid p_{j}(r, \nu)<0\right\} .
$$

If $\mathcal{I} \neq \emptyset$, then $\mathcal{I}$ is an open interval, and for any $r \in \mathcal{I}$, the ball $B_{\bar{x}}(r)$ contains a unique solution $\tilde{x}$ such that $F(\tilde{x})=0$. Note that $\tilde{x}$ is the same solution for all $r \in \mathcal{I}$.

We now derive the bounds $Y_{j}$ and $Z_{j}(r)$ for the definition of the radii polynomials which are defined in (2.15). Denote by $\bar{a}$ the solution we found using Newton's method.

Recall that the bounds $Y_{j}$ satisfy $\left\|[T(\bar{a})-\bar{a}]_{j}\right\|_{\nu} \leq Y_{j}$, where $T$ is our Newton-like operator. Recalling the action of each component of $A$ given in (2.13), a direct computation yields the following computable bounds.

$$
\begin{aligned}
& Y_{1} \stackrel{\text { def }}{=} \sum_{k=0}^{m-1}\left|\sum_{i=1}^{6}\left[A_{1, a_{i}}^{(m)} F_{i}^{(m)}(\bar{a})\right]_{k}\right| \nu^{k} \\
& Y_{2} \stackrel{\text { def }}{=} \sum_{k=0}^{m-1}\left|\sum_{i=1}^{6}\left[A_{2, a_{i}}^{(m)} F_{i}^{(m)}(\bar{a})\right]_{k}\right| \nu^{k}+\sum_{k=m}^{4 m-4} \frac{1}{k \omega}\left|\left[F_{2}(\bar{a})\right]_{k}\right| \nu^{k} \\
& Y_{3} \stackrel{\text { def }}{=} \sum_{k=0}^{m-1}\left|\sum_{i=1}^{6}\left[A_{3, a_{i}}^{(m)} F_{i}^{(m)}(\bar{a})\right]_{k}\right| \nu^{k} \\
& Y_{4} \stackrel{\text { def }}{=} \sum_{k=0}^{m-1}\left|\sum_{i=1}^{6}\left[A_{4, a_{i}}^{(m)} F_{i}^{(m)}(\bar{a})\right]_{k}\right| \nu^{k}+\sum_{k=m}^{4 m-4} \frac{1}{k \omega}\left|\left[F_{4}(\bar{a})\right]_{k}\right| \nu^{k} \\
& Y_{5} \stackrel{\text { def }}{=} \sum_{k=0}^{m-1}\left|\sum_{i=1}^{6}\left[A_{5, a_{i}}^{(m)} F_{i}^{(m)}(\bar{a})\right]_{k}\right| \nu^{k}+\sum_{k=m}^{5 m-5} \frac{1}{k \omega}\left|\left[F_{5}(\bar{a})\right]_{k}\right| \nu^{k} \\
& Y_{6} \stackrel{\text { def }}{=} \sum_{k=0}^{m-1}\left|\sum_{i=1}^{6}\left[A_{6, a_{i}}^{(m)} F_{i}^{(m)}(\bar{a})\right]_{k}\right| \nu^{k}+\sum_{k=m}^{5 m-5} \frac{1}{k \omega}\left|\left[F_{6}(\bar{a})\right]_{k}\right| \nu^{k} .
\end{aligned}
$$

To compute the bounds $Z_{1}(r), \ldots, Z_{6}(r)$, we have to bound each component of $D T(\bar{a}+b) c$, for $b, c \in B(r)$. Recall that

$$
D T(\bar{a}+b) c=\left(I-A A^{\dagger}\right) c-A\left(D F(\bar{a}+b)-A^{\dagger}\right) c
$$

where $A^{\dagger}$ is the approximation of $D F(\bar{a})$, as defined in Section 2.4. Let $B \stackrel{\text { def }}{=} I-A A^{\dagger}$. As the operator $A$ defined in Section 2.4, $B$ has the form $B=\left\{B_{j, a_{i}}\right\}_{i, j=1}^{6}$. Note that given $c \in X,\left((B c)_{j}\right)_{k}=0$ for all $k \geq m$ (e.g. see [1] for more 
details). By denoting $b=\tilde{b} r$ and $c=\tilde{c} r$ with $\tilde{b}, \tilde{c} \in B_{0}(1)$, we can deduce that for $j=1, \ldots, 6$, we have

$$
\left\|(B c)_{j}\right\|_{\nu}=\left\|(B \tilde{c})_{j}\right\|_{\nu} r \leq\left(\sum_{i=1}^{6}\left\|B_{j, a_{i}}\right\|_{B\left(\ell_{\nu}^{1}, \ell_{\nu}^{1}\right)}\right) r=Z_{j}^{(0)} r \stackrel{\text { def }}{=}\left(\sum_{i=1}^{6} K_{B, j, i}\right) r
$$

where

$$
K_{B, j, i} \stackrel{\text { def }}{=} \max _{0 \leq n \leq m-1} \frac{1}{\nu^{n}} \sum_{k=0}^{m-1}\left|\left(B_{j, a_{i}}\right)_{k, n}\right| \nu^{k} .
$$

Note that $K_{B, j, i}$ is in fact the norm $\left\|B_{j, a_{i}}\right\|_{B\left(\ell_{\nu}^{1}, \ell_{\nu}^{1}\right)}$ for operators of this (finite) form, that is for operators satisfying $\left(B_{j, a_{i}}\right)_{k, n}=0$ for all $k \geq m$ or $n \geq m$. Next, we need to bound $\left\|\left[-A\left(D F(\bar{a}+b)-A^{\dagger}\right) c\right]_{j}\right\|_{\nu}$, for $j=1, \ldots, 6$. For $k \geq 1$,

$$
\begin{aligned}
{[D F(\bar{a}+b) c]_{1} } & =k \omega\left(c_{1}\right)_{k}+\left(c_{2}\right)_{k} \\
{[D F(\bar{a}+b) c]_{2} } & =k \omega\left(c_{2}\right)_{k}+2\left(c_{4}\right)_{k}+\left(c_{1}\right)_{k}-(1-\mu)\left[c_{1}\left(\bar{a}_{5}+b_{5}\right)^{3}+3 c_{5}\left(\bar{a}_{1}+b_{1}\right)\left(\bar{a}_{5}+b_{5}\right)^{2}\right]_{k} \\
& -\left(\mu-\mu^{2}\right)\left[3 c_{5}\left(\bar{a}_{5}+b_{5}\right)^{2}\right]_{k}-\mu\left[c_{1}\left(\bar{a}_{6}+b_{6}\right)^{3}+3 c_{6}\left(\bar{a}_{1}+b_{1}\right)\left(\bar{a}_{6}+b_{6}\right)^{2}\right]_{k} \\
& +\left(\mu-\mu^{2}\right)\left[3 c_{6}\left(\bar{a}_{6}+b_{6}\right)^{2}\right]_{k} \\
{[D F(\bar{a}+b) c]_{3} } & =k \omega\left(c_{3}\right)_{k}+\left(c_{4}\right)_{k} \\
{[D F(\bar{a}+b) c]_{4} } & =k \omega\left(c_{4}\right)_{k}+2\left(c_{2}\right)_{k}-\left(c_{3}\right)_{k}+(1-\mu)\left[c_{3}\left(\bar{a}_{5}+b_{5}\right)^{3}+3 c_{5}\left(\bar{a}_{3}+b_{3}\right)\left(\bar{a}_{5}+b_{5}\right)^{2}\right]_{k} \\
& +\mu\left[c_{3}\left(\bar{a}_{6}+b_{6}\right)^{3}+3 c_{6}\left(\bar{a}_{3}+b_{3}\right)\left(\bar{a}_{6}+b_{6}\right)^{2}\right]_{k} \\
{[D F(\bar{a}+b) c]_{5} } & =k \omega\left(c_{5}\right)_{k}+\left[c_{1}\left(\bar{a}_{2}+b_{2}\right)\left(\bar{a}_{5}+b_{5}\right)^{3}+c_{2}\left(\bar{a}_{1}+b_{1}\right)\left(\bar{a}_{5}+b_{5}\right)^{3}\right. \\
& +3 c_{5}\left(\bar{a}_{1}+b_{1}\right)\left(\bar{a}_{2}+b_{2}\right)\left(\bar{a}_{5}+b_{5}\right)^{2}+c_{3}\left(\bar{a}_{4}+b_{4}\right)\left(\bar{a}_{5}+b_{5}\right)^{3} \\
& \left.+c_{4}\left(\bar{a}_{3}+b_{3}\right)\left(\bar{a}_{5}+b_{5}\right)^{3}+3 c_{5}\left(\bar{a}_{3}+b_{3}\right)\left(\bar{a}_{4}+b_{4}\right)\left(\bar{a}_{5}+b_{5}\right)^{2}\right]_{k} \\
& +\mu\left[c_{2}\left(\bar{a}_{5}+b_{5}\right)^{3}+3 c_{5}\left(\bar{a}_{2}+b_{2}\right)\left(\bar{a}_{5}+b_{5}\right)^{2}\right]_{k} \\
{[D F(\bar{a}+b) c]_{6} } & =k \omega\left(c_{6}\right)_{k}+\left[c_{1}\left(\bar{a}_{2}+b_{2}\right)\left(\bar{a}_{6}+b_{6}\right)^{3}+c_{2}\left(\bar{a}_{1}+b_{1}\right)\left(\bar{a}_{6}+b_{6}\right)^{3}\right. \\
& +3 c_{6}\left(\bar{a}_{1}+b_{1}\right)\left(\bar{a}_{2}+b_{2}\right)\left(\bar{a}_{6}+b_{6}\right)^{2}+c_{3}\left(\bar{a}_{4}+b_{4}\right)\left(\bar{a}_{6}+b_{6}\right)^{3} \\
& \left.+c_{4}\left(\bar{a}_{3}+b_{3}\right)\left(\bar{a}_{6}+b_{6}\right)^{3}+3 c_{6}\left(\bar{a}_{3}+b_{3}\right)\left(\bar{a}_{4}+b_{4}\right)\left(\bar{a}_{6}+b_{6}\right)^{2}\right]_{k} \\
& +(\mu-1)\left[c_{2}\left(\bar{a}_{6}+b_{6}\right)^{3}+3 c_{6}\left(\bar{a}_{2}+b_{2}\right)\left(\bar{a}_{6}+b_{6}\right)^{2}\right]_{k}
\end{aligned}
$$

and

$$
\begin{aligned}
\left(A^{\dagger} c\right)_{1} & =k \omega\left(c_{1}\right)_{k}+\left\{c_{2}\right\}_{k<m} \\
\left(A^{\dagger} c\right)_{2} & =k \omega\left(c_{2}\right)_{k}+\left\{2\left(c_{4}\right)+\left(c_{1}\right)-(1-\mu)\left(c_{1} \bar{a}_{5}^{3}\right)-3(1-\mu)\left(c_{5} \bar{a}_{1} \bar{a}_{5}^{2}\right)\right. \\
& \left.-3\left(\mu-\mu^{2}\right)\left(c_{5} \bar{a}_{5}^{2}\right)-\mu\left(c_{1} \bar{a}_{6}^{3}\right)-3 \mu\left(c_{6} \bar{a}_{1} \bar{a}_{6}^{2}\right)+3\left(\mu-\mu^{2}\right)\left(c_{6} \bar{a}_{6}^{2}\right)\right\}_{k<m} \\
\left(A^{\dagger} c\right)_{3} & =k \omega\left(c_{3}\right)_{k}+\left\{c_{4}\right\}_{k<m} \\
\left(A^{\dagger} c\right)_{4} & =k \omega\left(c_{4}\right)_{k}+\left\{2\left(c_{2}\right)-\left(c_{3}\right)+(1-\mu)\left(c_{3} \bar{a}_{5}^{3}\right)+3(1-\mu)\left(c_{5} \bar{a}_{3} \bar{a}_{5}^{2}\right)\right. \\
& \left.+\mu\left(c_{3} \bar{a}_{6}^{3}\right)+3 \mu\left(c_{6} \bar{a}_{3} \bar{a}_{6}^{2}\right)\right\}_{k<m} \\
\left(A^{\dagger} c\right)_{5} & =k \omega\left(c_{5}\right)_{k}+\left\{\left(c_{1} \bar{a}_{2} \bar{a}_{5}^{3}\right)+\left(c_{2} \bar{a}_{1} \bar{a}_{5}^{3}\right)+3\left(c_{5} \bar{a}_{1} \bar{a}_{2} \bar{a}_{5}^{2}\right)+\left(c_{3} \bar{a}_{4} \bar{a}_{5}^{3}\right)+\left(c_{4} \bar{a}_{3} \bar{a}_{5}^{3}\right)\right. \\
& \left.+3\left(c_{5} \bar{a}_{3} \bar{a}_{4} \bar{a}_{5}^{2}\right)+\mu\left(c_{2} \bar{a}_{5}^{3}\right)+3 \mu\left(c_{5} \bar{a}_{2} \bar{a}_{5}^{2}\right)\right\}_{k<m} \\
\left(A^{\dagger} c\right)_{6} & =k \omega\left(c_{6}\right)_{k}+\left\{\left(c_{1} \bar{a}_{2} \bar{a}_{6}^{3}\right)+\left(c_{2} \bar{a}_{1} \bar{a}_{6}^{3}\right)+3\left(c_{6} \bar{a}_{1} \bar{a}_{2} \bar{a}_{6}^{2}\right)+\left(c_{3} \bar{a}_{4} \bar{a}_{6}^{3}\right)+\left(c_{4} \bar{a}_{3} \bar{a}_{6}^{3}\right)\right. \\
& \left.+3\left(c_{6} \bar{a}_{3} \bar{a}_{4} \bar{a}_{6}^{2}\right)+(\mu-1)\left(c_{2} \bar{a}_{6}^{3}\right)+3(\mu-1)\left(c_{6} \bar{a}_{2} \bar{a}_{6}^{2}\right)\right\}_{k<m}
\end{aligned}
$$

From the definitions of $\left(F_{1}\right)_{0},\left(F_{3}\right)_{0}$ and $\left(F_{4}\right)_{0}$ in $(2.7)$ (which are linear), we get that $\left([D F(\bar{a}+b) c]_{j}-\left(A^{\dagger} c\right)_{j}\right)_{0}=0$ for 
$k=0$ and for $j=1,3,4$. Also,

$$
\begin{aligned}
\left([D F(\bar{a}+b) c]_{5}-\left(A^{\dagger} c\right)_{5}\right)_{0} & =\left[ \pm\left(2 \sum_{k \geq m}\left(\tilde{c}_{5}\right)_{k}\right)\left(2 \sum_{k=0}^{m-1}\left(\bar{a}_{1}\right)_{k}+\mu\right) \pm\left(2 \sum_{k \geq m}\left(\tilde{c}_{1}\right)_{k}\right)\left(2 \sum_{k=0}^{m-1}\left(\bar{a}_{1}\right)_{k}\right)\right] r \\
& +\left[ \pm\left(\left(\tilde{c}_{5}\right)_{0}+2 \sum_{k \geq 1}\left(\tilde{c}_{5}\right)_{k}\right)\left(\left(\tilde{b}_{1}\right)_{0}+2 \sum_{k \geq 1}\left(\tilde{b}_{1}\right)_{k}\right) \pm\left(\left(\tilde{b}_{5}\right)_{0}+2 \sum_{k \geq 1}\left(\tilde{b}_{5}\right)_{k}\right)\left(\left(\tilde{c}_{1}\right)_{0}+2 \sum_{k \geq 1}\left(\tilde{c}_{1}\right)_{k}\right)\right] r^{2},
\end{aligned}
$$

where the signs \pm depend on the cases defining $\eta_{1}$ and $\eta_{2}$ in (2.8) and (2.9), respectively. Now, for $a \in \ell_{\nu}^{1}$ such that $\|a\|_{\nu} \leq 1$, we have that

$$
\left|a_{0}+2 \sum_{k \geq 1} a_{k}\right| \leq 2 \sum_{k \geq 0}\left|a_{k}\right| \leq 2 \sum_{k \geq 0}\left|a_{k}\right| \nu^{k}=2\|a\|_{\nu} \leq 2 .
$$

Hence,

$$
\begin{aligned}
& \left|\left([D F(\bar{a}+b) c]_{5}-\left(A^{\dagger} c\right)_{5}\right)_{0}\right| \leq \frac{2}{\nu^{m}}\left(\left|2 \sum_{k=0}^{m-1}\left(\bar{a}_{1}\right)_{k}+\mu\right|+\left|2 \sum_{k=0}^{m-1}\left(\bar{a}_{1}\right)_{k}\right|\right) r+8 r^{2} \\
& \left|\left([D F(\bar{a}+b) c]_{6}-\left(A^{\dagger} c\right)_{6}\right)_{0}\right| \leq \frac{2}{\nu^{m}}\left(\left|2 \sum_{k=0}^{m-1}\left(\bar{a}_{1}\right)_{k}+\mu-1\right|+\left|2 \sum_{k=0}^{m-1}\left(\bar{a}_{1}\right)_{k}\right|\right) r+8 r^{2} .
\end{aligned}
$$

Given an element $a \in \ell_{\nu}^{1}$, denote by $a_{F}$ the finite-dimensional vector $a_{F}=\left(a_{0}, a_{1}, \ldots, a_{m-1}\right)^{T} \in \mathbb{R}^{m}$.

We now use the triangle inequality and Lemma 2 to bound $\left\|\left[-A\left(D F(\bar{a}+b)-A^{\dagger}\right) c\right]_{j}\right\|_{\nu}$. By substituting $b$ and $c$ with $\tilde{b} r$ and $\tilde{c} r$, and grouping the corresponding powers of $r$ together, we obtain that

$$
\begin{aligned}
& Z_{j}^{(1)} \stackrel{\text { def }}{=} \frac{2}{\nu^{m}}\left\|\left(A_{j, a_{5}}\right)_{:, 0}\right\|_{\nu}\left(\left|2 \sum_{k=0}^{m-1}\left(\bar{a}_{1}\right)_{k}+\mu\right|+\left|2 \sum_{k=0}^{m-1}\left(\bar{a}_{1}\right)_{k}\right|\right) \\
& +\frac{2}{\nu^{m}}\left\|\left(A_{j, a_{6}}\right)_{:, 0}\right\|_{\nu}\left(\left|2 \sum_{k=0}^{m-1}\left(\bar{a}_{1}\right)_{k}+\mu-1\right|+\left|2 \sum_{k=0}^{m-1}\left(\bar{a}_{1}\right)_{k}\right|\right) \\
& +\frac{6}{m \omega}+|\mu-1|||\left|A_{j, a_{2}}\right|\left[\left|\bar{a}_{5}\right|^{3} w\right]_{F}\left\|_{\nu}+3|\mu-1|||\left|A_{j, a_{2}}\right|\left[\left|\bar{a}_{1}\right|\left|\bar{a}_{5}\right|^{2} w\right]_{F}\right\|_{\nu} \\
& +3\left|\mu^{2}-\mu\right|\left\|\left|A_{j, a_{2}}\right|\left[\left|\bar{a}_{5}\right|^{2} w\right]_{F}\right\|_{\nu}+\mu\left\||| A_{j, a_{2}}\left|\left[\left|\bar{a}_{6}\right|^{3} w\right]_{F}\left\|_{\nu}+3 \mu\right\|\right|\left|A_{j, a_{2}}\right|\left[\left|\bar{a}_{1}\right|\left|\bar{a}_{6}\right|^{2} w\right]_{F}\right\|_{\nu} \\
& +3\left|\mu^{2}-\mu\right|||\left|A_{j, a_{2}}\right|\left[\left|\bar{a}_{6}\right|^{2} w\right]_{F}\left\|_{\nu}+|\mu-1|||\left|A_{j, a_{4}}\right|\left[\left|\bar{a}_{5}\right|^{3} w\right]_{F}\right\|_{\nu} \\
& +3|\mu-1|||\left|A_{j, a_{4}}\right|\left[\left|\bar{a}_{3}\right|\left|\bar{a}_{5}\right|^{2} w\right]_{F}\left\|_{\nu}+\mu\right\||| A_{j, a_{4}}\left|\left[\left|\bar{a}_{6}\right|^{3} w\right]_{F}\left\|_{\nu}+3 \mu\right\|\right|\left|A_{j, a_{4}}\right|\left[\left|\bar{a}_{3}\right|\left|\bar{a}_{6}\right|^{2} w\right]_{F} \|_{\nu} \\
& +\left\|\left|A_{j, a_{5}}\right|\left[\left|\bar{a}_{1}\right|\left|\bar{a}_{5}\right|^{3} w\right]_{F}\right\|_{\nu}+\left\|\left|A_{j, a_{5}}\right|\left[\left|\bar{a}_{2}\right|\left|\bar{a}_{5}\right|^{3} w\right]_{F}\right\|_{\nu}+\left\|\left|A_{j, a_{5}}\right|\left[\left|\bar{a}_{3}\right|\left|\bar{a}_{5}\right|^{3} w\right]_{F}\right\|_{\nu} \\
& +||\left|A_{j, a_{5}}\right|\left[\left|\bar{a}_{4}\right|\left|\bar{a}_{5}\right|^{3} w\right]_{F}\left\|_{\nu}+3\right\|\left|A_{j, a_{5}}\right|\left[\left|\bar{a}_{1}\right|\left|\bar{a}_{2}\right|\left|\bar{a}_{5}\right|^{2} w\right]_{F} \|_{\nu} \\
& +3\left\|\left|A_{j, a_{5}}\right|\left[\left|\bar{a}_{3}\right|\left|\bar{a}_{4}\right|\left|\bar{a}_{5}\right|^{2} w\right]_{F}\right\|_{\nu}+\mu\left\||| A_{j, a_{5}}\left|\left[\left|\bar{a}_{5}\right|^{3} w\right]_{F}\left\|_{\nu}+3 \mu\right\|\right|\left|A_{j, a_{5}}\right|\left[\left|\bar{a}_{2}\right|\left|\bar{a}_{5}\right|^{2} w\right]_{F}\right\|_{\nu} \\
& +\left\|\left|A_{j, a_{6}}\right|\left[\left|\bar{a}_{1}\right|\left|\bar{a}_{6}\right|^{3} w\right]_{F}\right\|_{\nu}+\left\|\left|A_{j, a_{6}}\right|\left[\left|\bar{a}_{2}\right|\left|\bar{a}_{6}\right|^{3} w\right]_{F}\right\|_{\nu}+\left\|\left|A_{j, a_{6}}\right|\left[\left|\bar{a}_{3}\right|\left|\bar{a}_{6}\right|^{3} w\right]_{F}\right\|_{\nu} \\
& +\left\|\left|A_{j, a_{6}}\right|\left[\left|\bar{a}_{4}\right|\left|\bar{a}_{6}\right|^{3} w\right]_{F}\right\|_{\nu}+3\left\|\left|A_{j, a_{6}}\right|\left[\left|\bar{a}_{1}\right|\left|\bar{a}_{2}\right|\left|\bar{a}_{6}\right|^{2} w\right]_{F}\right\|_{\nu} \\
& +3\left\|\left|A_{j, a_{6}}\right|\left[\left|\bar{a}_{3}\right|\left|\bar{a}_{4}\right|\left|\bar{a}_{6}\right|^{2} w\right]_{F}\right\|_{\nu}+|\mu-1|\left\|\left|A_{j, a_{6}}\right|\left[\left|\bar{a}_{6}\right|^{3} w\right]_{F}\right\|_{\nu} \\
& +3|\mu-1|||\left|A_{j, a_{6}}\right|\left[\left|\bar{a}_{2}\right|\left|\bar{a}_{6}\right|^{2} w\right]_{F} \|_{\nu},
\end{aligned}
$$


where $\left(A_{j, a_{i}}\right)_{:, 0} \in \ell_{\nu}^{1}$ is the first column of the operator for $i=5,6$, and where $w \stackrel{\text { def }}{=}\left(0,0, \ldots, 0, \frac{1}{\nu^{m}}, \frac{1}{\nu^{m+1}}, \frac{1}{\nu^{m+2}}, \ldots\right)$.

$$
\begin{aligned}
& Z_{j}^{(2)} \stackrel{\text { def }}{=} 8\left(\left\|\left(A_{j, a_{5}}\right)_{:, 0}\right\|_{\nu}+\left\|\left(A_{j, a_{6}}\right)_{:, 0}\right\|_{\nu}\right) \\
& +4\left\|A_{j, a_{2}}\right\|\left[|\mu-1|\left(24\left\|\bar{a}_{5}\right\|_{\nu}^{2}+24\left\|\bar{a}_{1}\right\|_{\nu}\left\|\bar{a}_{5}\right\|_{\nu}\right)+6\left|\mu^{2}-\mu\right|\left\|\bar{a}_{5}\right\|_{\nu}\right. \\
& \left.+24 \mu\left(\left\|\bar{a}_{6}\right\|_{\nu}^{2}+\left\|\bar{a}_{1}\right\|_{\nu}\left\|\bar{a}_{6}\right\|_{\nu}\right)+6\left|\mu^{2}-\mu\right|\left\|\bar{a}_{6}\right\|_{\nu}\right] \\
& +4\left\|A_{j, a_{4}}\right\|\left[|\mu-1|\left(24\left\|\bar{a}_{5}\right\|_{\nu}^{2}+24\left\|\bar{a}_{3}\right\|_{\nu}\left\|\bar{a}_{5}\right\|_{\nu}\right)+24 \mu\left(\left\|\bar{a}_{6}\right\|_{\nu}^{2}+\left\|\bar{a}_{3}\right\|_{\nu}\left\|\bar{a}_{6}\right\|_{\nu}\right)\right] \\
& +4\left\|A_{j, a_{5}}\right\|\left[64\left\|\bar{a}_{5}\right\|_{\nu}^{3}+24\left\|\bar{a}_{5}\right\|_{\nu}^{2}\left(4\left\|\bar{a}_{1}\right\|_{\nu}+4\left\|\bar{a}_{2}\right\|_{\nu}+4\left\|\bar{a}_{3}\right\|_{\nu}+4\left\|\bar{a}_{4}\right\|_{\nu}+\mu\right)\right. \\
& \left.+24\left\|\bar{a}_{5}\right\|_{\nu}\left(\mu\left\|\bar{a}_{2}\right\|_{\nu}+4\left\|\bar{a}_{1}\right\|_{\nu}\left\|\bar{a}_{2}\right\|_{\nu}+4\left\|\bar{a}_{3}\right\|_{\nu}\left\|\bar{a}_{4}\right\|_{\nu}\right)\right] \\
& +4\left\|A_{j, a_{6}}\right\|\left[64\left\|\bar{a}_{6}\right\|_{\nu}^{3}+24\left\|\bar{a}_{6}\right\|_{\nu}^{2}\left(4\left\|\bar{a}_{1}\right\|_{\nu}+4\left\|\bar{a}_{2}\right\|_{\nu}+4\left\|\bar{a}_{3}\right\|_{\nu}+4\left\|\bar{a}_{4}\right\|_{\nu}+|\mu-1|\right)\right. \\
& \left.+24\left\|\bar{a}_{6}\right\|_{\nu}\left(|\mu-1|\left\|\bar{a}_{2}\right\|_{\nu}+4\left\|\bar{a}_{1}\right\|_{\nu}\left\|\bar{a}_{2}\right\|_{\nu}+4\left\|\bar{a}_{3}\right\|_{\nu}\left\|\bar{a}_{4}\right\|_{\nu}\right)\right] \text {, } \\
& Z_{j}^{(3)} \stackrel{\text { def }}{=} 16\left\|A_{j, a_{2}}\right\|\left[9|\mu-1|\left\|\bar{a}_{5}\right\|_{\nu}+9 \mu\left\|\bar{a}_{6}\right\|_{\nu}+3|\mu-1|\left\|\bar{a}_{1}\right\|_{\nu}+3 \mu\left\|\bar{a}_{1}\right\|_{\nu}+3\left|\mu^{2}-\mu\right|\right] \\
& +16\left\|A_{j, a_{4}}\right\|\left[9|\mu-1|\left\|\bar{a}_{5}\right\|_{\nu}+9 \mu\left\|\bar{a}_{6}\right\|_{\nu}+3 \mu\left\|\bar{a}_{3}\right\|_{\nu}\right] \\
& +16\left\|A_{j, a_{5}}\right\|\left[72\left\|\bar{a}_{5}\right\|_{\nu}^{2}+9\left\|\bar{a}_{5}\right\|_{\nu}\left(4\left\|\bar{a}_{1}\right\|_{\nu}+4\left\|\bar{a}_{2}\right\|_{\nu}+4\left\|\bar{a}_{3}\right\|_{\nu}+4\left\|\bar{a}_{4}\right\|_{\nu}+\mu\right)\right. \\
& \left.+3 \mu\left\|\bar{a}_{2}\right\|_{\nu}+12\left\|\bar{a}_{1}\right\|_{\nu}\left\|\bar{a}_{2}\right\|_{\nu}+12\left\|\bar{a}_{3}\right\|_{\nu}\left\|\bar{a}_{4}\right\|_{\nu}\right] \\
& +16\left\|A_{j, a_{6}}\right\|\left[72\left\|\bar{a}_{6}\right\|_{\nu}^{2}+9\left\|\bar{a}_{6}\right\|_{\nu}\left(4\left\|\bar{a}_{1}\right\|_{\nu}+4\left\|\bar{a}_{2}\right\|_{\nu}+4\left\|\bar{a}_{3}\right\|_{\nu}+4\left\|\bar{a}_{4}\right\|_{\nu}+|\mu-1|\right)\right. \\
& \left.+3|\mu-1|\left\|\bar{a}_{2}\right\|_{\nu}+12\left\|\bar{a}_{1}\right\|_{\nu}\left\|\bar{a}_{2}\right\|_{\nu}+12\left\|\bar{a}_{3}\right\|_{\nu}\left\|\bar{a}_{4}\right\|_{\nu}\right] \text {, } \\
& Z_{j}^{(4)} \stackrel{\text { def }}{=} 64|8 \mu-1||| A_{j, a_{2}}\left\|+64|8 \mu-1||| A_{j, a_{4}}\right\| \\
& +64\left\|A_{j, a_{5}}\right\|\left[24\left\|\bar{a}_{5}\right\|_{\nu}+4\left\|\bar{a}_{1}\right\|_{\nu}+4\left\|\bar{a}_{2}\right\|_{\nu}+4\left\|\bar{a}_{3}\right\|_{\nu}+4\left\|\bar{a}_{4}\right\|_{\nu}+4 \mu\right] \\
& +64\left\|A_{j, a_{6}}\right\|\left[24\left\|\bar{a}_{6}\right\|_{\nu}+4\left\|\bar{a}_{1}\right\|_{\nu}+4\left\|\bar{a}_{2}\right\|_{\nu}+4\left\|\bar{a}_{3}\right\|_{\nu}+4\left\|\bar{a}_{4}\right\|_{\nu}+4|\mu-1|\right], \\
& Z_{j}^{(5)} \stackrel{\text { def }}{=} 2560\left(\left\|A_{j, a_{5}}\right\|+\left\|A_{j, a_{6}}\right\|\right) .
\end{aligned}
$$

Combining (2.18), (2.19), (2.20), (2.21), (2.22), (2.23), (2.24), (2.25), (2.26), (2.27) and (2.28), we define, for each $j=1, \ldots, 6$, the quintic radii polynomial $p_{j}(r)$ by

$$
p_{j}(r) \stackrel{\text { def }}{=} Z_{j}^{(5)} r^{5}+Z_{j}^{(4)} r^{4}+Z_{j}^{(3)} r^{3}+Z_{j}^{(2)} r^{2}+\left(Z_{j}^{(1)}-1\right) r+Y_{j}
$$

\subsection{Results}

We finally present some applications of the radii polynomial approach. Using computer programs in MATLAB together with the interval arithmetic toolbox INTLAB, we compute the radii polynomials $p_{1}(r), \ldots, p_{6}(r)$ given by $(2.29)$, and we apply Proposition 4 to prove existence of periodic solutions of the planar circular restricted 3-body problem (2.1). All codes are available at [93]. We proved the existence of periodic orbits in the case where the two large bodies have the same mass, that is the mass ratio is $\mu=\frac{1}{2}$. These orbits are shown in Figure 3. Moreover, we proved the existence of periodic orbits in the earth-moon system, that is with the mass ratio $\mu=0.0123$. These orbits are shown in Figure 4.

\section{References}

[1] Allan Hungria, Jean-Philippe Lessard, and Jason D. Mireles-James. Rigorous numerics for analytic solutions of differential equations: the radii polynomial approach. Math. Comp., September 2015. 


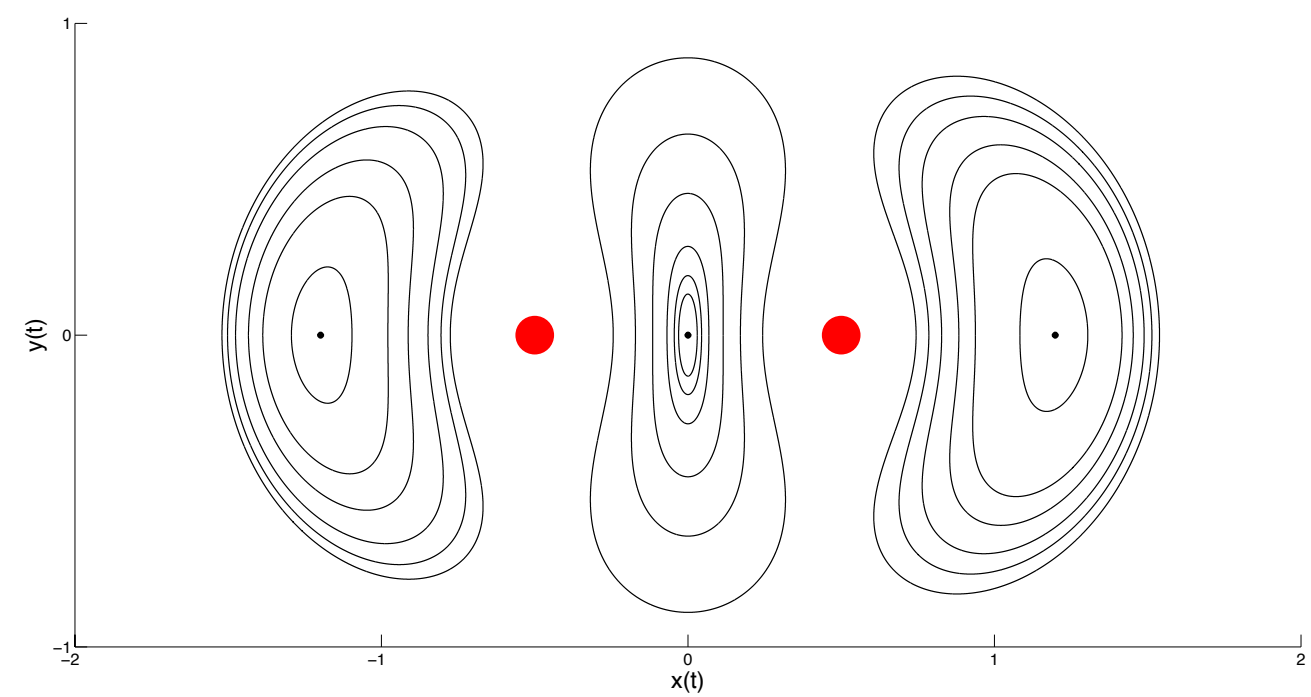

Figure 3: In this figure, we show the picture of several rigorously computed periodic orbits for the planar circular restricted 3-body problem (2.1). The code performing the proof of existence of all orbits in the figure is script_proof_figure_3.m which is available at [93]. The run time for doing all proofs is about 450 seconds on a MacBook Pro 2014 with Processor $3 \mathrm{GHz}$ Intel Core i7 and $8 \mathrm{~GB}$ of Memory. The red circles depict the location of the primaries, and the black dots on the $x$ axis to the left, center, and right of the primaries denote the equilibrium points. The mass ratio between the bodies is $\mu=0.5$ which corresponds to two bodies with equal mass. The largest periodic orbit of the left family has frequency $\omega \approx 1.276$, and is proven with 149 Fourier coefficients, $\nu=1.01$ and $r=2.9 \times 10^{-10}$. The largest periodic orbit of the center family has frequency $\omega \approx 1.283$, and is proven with 157 Fourier coefficients, $\nu=1.015$ and $r=4.5 \times 10^{-9}$. The largest periodic orbit of the right family has frequency $\omega \approx 1.286$, and is proven with 122 Fourier coefficients, $\nu=1.01$ and $r=2.5 \times 10^{-9}$.

[2] Sarah Day, Jean-Philippe Lessard, and Konstantin Mischaikow. Validated continuation for equilibria of PDEs. SIAM J. Numer. Anal., 45(4):1398-1424 (electronic), 2007.

[3] Marcio Gameiro, Jean-Philippe Lessard, and Alessandro Pugliese. Computation of smooth manifolds via rigorous multi-parameter continuation in infinite dimensions. Found. Comput. Math., June 2015.

[4] James M. Ortega. The Newton-Kantorovich theorem. Amer. Math. Monthly, 75:658-660, 1968.

[5] Hans Koch, Alain Schenkel, and Peter Wittwer. Computer-assisted proofs in analysis and programming in logic: a case study. SIAM Rev., 38(4):565-604, 1996.

[6] Nobito Yamamoto. A numerical verification method for solutions of boundary value problems with local uniqueness by Banach's fixed-point theorem. SIAM J. Numer. Anal., 35(5):2004-2013 (electronic), 1998.

[7] Zbigniew Galias and Piotr Zgliczyński. Infinite-dimensional Krawczyk operator for finding periodic orbits of discrete dynamical systems. Internat. J. Bifur. Chaos Appl. Sci. Engrg., 17(12):4261-4272, 2007.

[8] Jean-Philippe Lessard and Christian Reinhardt. Rigorous Numerics for Nonlinear Differential Equations Using Chebyshev Series. SIAM J. Numer. Anal., 52(1):1-22, 2014.

[9] Jan Bouwe van den Berg and Jean-Philippe Lessard. Chaotic braided solutions via rigorous numerics: chaos in the Swift-Hohenberg equation. SIAM J. Appl. Dyn. Syst., 7(3):988-1031, 2008. 


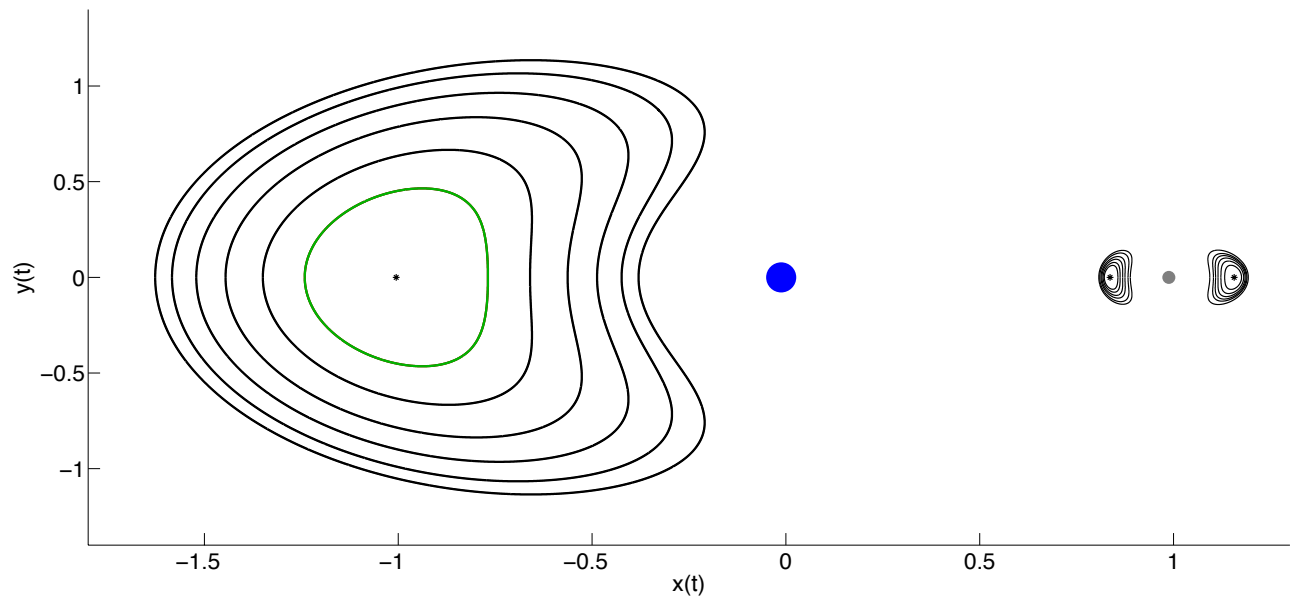

Figure 4: In this figure, we show the picture of several rigorously computed periodic orbits for the planar circular restricted 3-body problem (2.1). The blue circle depicts the Earth and the grey dot depicts the moon. The three black dots on the $x$-axis to the left, center, and right of the primaries denoted the equilibrium solutions. The mass ratio between the bodies is $\mu=0.0123$ which corresponds to the earth-moon mass ratio. (The sizes of the dots indicating the earth and the moon are not shown to scale). The largest periodic orbit of the left family has frequency $\omega \approx 1.0079$, and is proven with 130 Fourier coefficients, $\nu=1.012$ and $r=6.1 \times 10^{-9}$. The largest periodic orbit of the center family has frequency $\omega \approx 2.0614$, and is proven with 61 Fourier coefficients, $\nu=1.02$ and $r=1.75 \times 10^{-9}$. The largest periodic orbit of the right family has frequency $\omega \approx 1.7906$, and is proven with 58 Fourier coefficients, $\nu=1.013$ and $r=3.52 \times 10^{-10}$. The orbit in green is proven in Theorem 1. The code performing the proof of existence of the green orbit only is script_proof_theorem $1 . \mathrm{m}$ which is available at [93]. The run time for the proof of the green orbit is about 7 seconds on a MacBook Pro 2014 with Processor $3 \mathrm{GHz}$ Intel Core i7 and 8 GB of Memory.

[10] Marcio Gameiro and Jean-Philippe Lessard. Analytic estimates and rigorous continuation for equilibria of higherdimensional PDEs. J. Differential Equations, 249(9):2237-2268, 2010.

[11] Lorenzo D'Ambrosio, Jean-Philippe Lessard, and Alessandro Pugliese. Blow-up profile for solutions of a fourth order nonlinear equation. Nonlinear Anal., 121:280-335, 2015.

[12] Roberto Castelli and Jean-Philippe Lessard. Rigorous Numerics in Floquet Theory: Computing Stable and Unstable Bundles of Periodic Orbits. SIAM J. Appl. Dyn. Syst., 12(1):204-245, 2013.

[13] Jean-Philippe Lessard. Recent advances about the uniqueness of the slowly oscillating periodic solutions of Wright's equation. J. Differential Equations, 248(5):992-1016, 2010.

[14] Jan Bouwe van den Berg, J. D. Mireles James, and Christian Reinhardt. Computing (un)stable manifolds with validated error bounds: non-resonant and resonant spectra. Submitted., 2016.

[15] Anaïs Correc and Jean-Philippe Lessard. Coexistence of nontrivial solutions of the one-dimensional Ginzburg-Landau equation: a computer-assisted proof. European J. Appl. Math., 26(1):33-60, 2015.

[16] Roberto Castelli and Holger Teismann. Rigorous numerics for NLS: bound states, spectra, and controllability. Preprint, 2015.

[17] Jean-Philippe Lessard, Jason D. Mireles James, and Christian Reinhardt. Computer assisted proof of transverse saddle-to-saddle connecting orbits for first order vector fields. J. Dynam. Differential Equations, 26(2):267-313, 2014. 
[18] Jan Bouwe van den Berg, Jason D. Mireles-James, Jean-Philippe Lessard, and Konstantin Mischaikow. Rigorous numerics for symmetric connecting orbits: even homoclinics of the Gray-Scott equation. SIAM J. Math. Anal., 43(4):1557-1594, 2011.

[19] Jan Bouwe van den Berg, Andréa Deschênes, Jean-Philippe Lessard, and Jason D. Mireles James. Stationary coexistence of hexagons and rolls via rigorous computations. SIAM J. Appl. Dyn. Syst., 14(2):942-979, 2015.

[20] Donald E. Knuth. The art of computer programming. Vol. 2. Addison-Wesley Publishing Co., Reading, Mass., second edition, 1981. Seminumerical algorithms, Addison-Wesley Series in Computer Science and Information Processing.

[21] Erwin Fehlberg. Zur numerischen Integration von Differentialgleichungen durch Potenzreihen-Ansätze, dargestellt an Hand physikalischer Beispiele. Z. Angew. Math. Mech., 44:83-88, 1964.

[22] H. Martin Bücker and George F. Corliss. A bibliography of automatic differentiation. In Automatic differentiation: applications, theory, and implementations, volume 50 of Lect. Notes Comput. Sci. Eng., pages 321-322. Springer, Berlin, 2006.

[23] Àngel Jorba and Maorong Zou. A software package for the numerical integration of ODEs by means of high-order Taylor methods. Experiment. Math., 14(1):99-117, 2005.

[24] A. Haro. Automatic differentiation methods in computational dynamical systems: Invariant manifolds and normal forms of vector fields at fixed points. Manuscript.

[25] Warwick Tucker. Validated numerics. Princeton University Press, Princeton, NJ, 2011. A short introduction to rigorous computations.

[26] Martin Berz and Kyoko Makino. Verified integration of ODEs and flows using differential algebraic methods on high-order Taylor models. Reliab. Comput., 4(4):361-369, 1998.

[27] Kyoko Makino and Martin Berz. Taylor models and other validated functional inclusion methods. Int. J. Pure Appl. Math., 4(4):379-456, 2003.

[28] A. Wittig, M. Berz, J. Grote, K. Makino, and S. Newhouse. Rigorous and accurate enclosure of invariant manifolds on surfaces. Regul. Chaotic Dyn., 15(2-3):107-126, 2010.

[29] Piotr Zgliczynski. $C^{1}$ Lohner algorithm. Found. Comput. Math., 2(4):429-465, 2002.

[30] Daniel Wilczak and Piotr Zgliczynski. Heteroclinic connections between periodic orbits in planar restricted circular three-body problem - a computer assisted proof. Comm. Math. Phys., 234(1):37-75, 2003.

[31] Piotr Zgliczyński. Rigorous numerics for dissipative partial differential equations. II. Periodic orbit for the KuramotoSivashinsky PDE — a computer-assisted proof. Found. Comput. Math., 4(2):157-185, 2004.

[32] S.M. Rump. INTLAB - INTerval LABoratory. In Tibor Csendes, editor, Developments in Reliable Computing, pages 77-104. Kluwer Academic Publishers, Dordrecht, 1999. http://www.ti3.tu-harburg.de/rump/.

[33] R. de la Llave and D. Rana. Accurate strategies for K.A.M. bounds and their implementation. In Computer aided proofs in analysis (Cincinnati, OH, 1989), volume 28 of IMA Vol. Math. Appl., pages 127-146. Springer, New York, 1991.

[34] Kenneth R. Meyer and Dieter S. Schmidt, editors. Computer aided proofs in analysis, volume 28 of The IMA Volumes in Mathematics and its Applications. Springer-Verlag, New York, 1991.

[35] Gianni Arioli and Hans Koch. Integration of dissipative partial differential equations: a case study. SIAM J. Appl. Dyn. Syst., 9(3):1119-1133, 2010. 
[36] Sarah Day and William D. Kalies. Rigorous computation of the global dynamics of integrodifference equations with smooth nonlinearities. SIAM J. Numer. Anal., 51(6):2957-2983, 2013.

[37] Gianni Arioli, Vivina Barutello, and Susanna Terracini. A new branch of Mountain Pass solutions for the choreographical 3-body problem. Comm. Math. Phys., 268(2):439-463, 2006.

[38] Oscar E. Lanford, III. A computer-assisted proof of the Feigenbaum conjectures. Bull. Amer. Math. Soc. (N.S.), 6(3):427-434, 1982.

[39] Hans Koch. Existence of critical invariant tori. Ergodic Theory Dynam. Systems, 28(6):1879-1894, 2008.

[40] J.-P. Eckmann, H. Koch, and P. Wittwer. A computer-assisted proof of universality for area-preserving maps. Mem. Amer. Math. Soc., 47(289):vi+122, 1984.

[41] Denis Gaidashev and Tomas Johnson. Dynamics of the universal area-preserving map associated with period-doubling: stable sets. J. Mod. Dyn., 3(4):555-587, 2009.

[42] Denis G. Gaidashev. Cylinder renormalization for Siegel discs and a constructive measurable Riemann mapping theorem. Nonlinearity, 20(3):713-741, 2007.

[43] Oscar E. Lanford, III. Computer-assisted proofs in analysis. Phys. A, 124(1-3):465-470, 1984. Mathematical physics, VII (Boulder, Colo., 1983).

[44] Jason D. Mireles-James and Konstantin Mischaikow. Computational proofs in dynamics. Encyclopedia of Applied Computational Mathematics, 2015.

[45] Jan Bouwe van den Berg and Jean-Philippe Lessard. Rigorous numerics in dynamics. Notices of the American Mathematical Society,, 62(9):1057-1061, 2015.

[46] Konstantin Mischaikow and Marian Mrozek. Chaos in the Lorenz equations: a computer-assisted proof. Bull. Amer. Math. Soc. (N.S.), 32(1):66-72, 1995.

[47] Konstantin Mischaikow and Marian Mrozek. Chaos in the Lorenz equations: a computer assisted proof. II. Details. Math. Comp., 67(223):1023-1046, 1998.

[48] Z. Galias and P. Zgliczyński. Computer assisted proof of chaos in the Lorenz equations. Phys. D, 115(3-4):165-188, 1998.

[49] Warwick Tucker. A rigorous ODE Solver and Smale's 14th Problem. Foundations of Computational Mathematics, 2(1):53-117-117, 2002-12-21.

[50] Warwick Tucker. The Lorenz attractor exists. C. R. Acad. Sci. Paris Sér. I Math., 328(12):1197-1202, 1999.

[51] Tomasz Kapela and Piotr Zgliczyński. A Lohner-type algorithm for control systems and ordinary differential inclusions. Discrete Contin. Dyn. Syst. Ser. B, 11(2):365-385, 2009.

[52] Piotr Zgliczyński. Rigorous numerics for dissipative PDEs III. An effective algorithm for rigorous integration of dissipative PDEs. Topol. Methods Nonlinear Anal., 36(2):197-262, 2010.

[53] Jacek Cyranka. Efficient and generic algorithm for rigorous integration forward in time of dPDEs: Part I. J. Sci. Comput., 59(1):28-52, 2014.

[54] S. Day, O. Junge, and K. Mischaikow. A rigorous numerical method for the global analysis of infinite-dimensional discrete dynamical systems. SIAM J. Appl. Dyn. Syst., 3(2):117-160 (electronic), 2004.

[55] Tadeusz Ważewski. Sur un principe topologique de l'examen de l'allure asymptotique des intégrales des équations différentielles ordinaires. Ann. Soc. Polon. Math., 20:279-313 (1948), 1947. 
[56] Dietmar Salamon. Morse theory, the Conley index and Floer homology. Bull. London Math. Soc., 22(2):113-140, 1990.

[57] Richard McGehee. The stable manifold theorem via an isolating block. In Symposium on Ordinary Differential Equations (Univ. Minnesota, Minneapolis, Minn., 1972; dedicated to Hugh L. Turrittin), pages 135-144. Lecture Notes in Math., Vol. 312. Springer, Berlin, 1973.

[58] Maciej J. Capiński and Piotr Zgliczyński. Geometric proof for normally hyperbolic invariant manifolds. J. Differential Equations, 259(11):6215-6286, 2015.

[59] Erik Boczko, William D. Kalies, and Konstantin Mischaikow. Polygonal approximation of flows. Topology Appl., 154(13):2501-2520, 2007.

[60] Marian Mrozek and Roman Srzednicki. Topological approach to rigorous numerics of chaotic dynamical systems with strong expansion of error bounds. Found. Comput. Math., 10(2):191-220, 2010.

[61] Sarah Day, Yasuaki Hiraoka, Konstantin Mischaikow, and Toshi Ogawa. Rigorous numerics for global dynamics: a study of the Swift-Hohenberg equation. SIAM J. Appl. Dyn. Syst., 4(1):1-31 (electronic), 2005.

[62] Thomas Stephens and Thomas Wanner. Rigorous validation of isolating blocks for flows and their Conley indices. SIAM J. Appl. Dyn. Syst., 13(4):1847-1878, 2014.

[63] Maciej Capiński and Anna Wasieczko. Geometric proof of strong stable/unstable manifolds, with applications to the restricted three body problem. Topoligical methods in nonlinear analysis, 46(1):363-399, 2015.

[64] Lamberto Cesari. Functional analysis and periodic solutions of nonlinear differential equations. Contributions to Differential Equations, 1:149-187, 1963.

[65] Lamberto Cesari. Functional analysis and Galerkin's method. Michigan Math. J., 11:385-414, 1964.

[66] Gianni Arioli and Hans Koch. Non-symmetric low-index solutions for a symmetric boundary value problem. $J$. Differential Equations, 252(1):448-458, 2012.

[67] Gábor Kiss and Jean-Philippe Lessard. Computational fixed-point theory for differential delay equations with multiple time lags. J. Differential Equations, 252(4):3093-3115, 2012.

[68] Roberto Castelli, Marcio Gameiro, and Jean-Philippe Lessard. Rigorous numerics for ill-posed pdes: periodic orbits in the boussinesq equation. Submitted, 2016.

[69] X. Cabré, E. Fontich, and R. de la Llave. The parameterization method for invariant manifolds. III. Overview and applications. J. Differential Equations, 218(2):444-515, 2005.

[70] Antoni Guillamon and Gemma Huguet. A computational and geometric approach to phase resetting curves and surfaces. SIAM J. Appl. Dyn. Syst., 8(3):1005-1042, 2009.

[71] Roberto Castelli, Jean-Philippe Lessard, and J. D. Mireles James. Parameterization of Invariant Manifolds for Periodic Orbits I: Efficient Numerics via the Floquet Normal Form. SIAM J. Appl. Dyn. Syst., 14(1):132-167, 2015.

[72] Amadeu Delshams, Rafael de la Llave, and Tere M. Seara. Geometric properties of the scattering map of a normally hyperbolic invariant manifold. Adv. Math., 217(3):1096-1153, 2008.

[73] Héctor E. Lomelí and James D. Meiss. Heteroclinic primary intersections and codimension one Melnikov method for volume-preserving maps. Chaos, 10(1):109-121, 2000. Chaotic kinetics and transport (New York, 1998).

[74] Jordi-Lluís Figueras, Àlex Haro, and A. Luque. Rigorous computer assisted application of kam theory: a modern approach. (Submitted), 2016. 
[75] Jordi-Lluís Figueras and Àlex Haro. Reliable computation of robust response tori on the verge of breakdown. SIAM J. Appl. Dyn. Syst., 11(2):597-628, 2012.

[76] Àlex. Haro, M. Canadell, Jordi-Lluís. Figueras, A. Luque, and J-M. Mondelo. The parameterization method for invariant manifolds: from theory to effective computations. Preprint http://www.maia.ub.es/ alex, 2014.

[77] Alessandra Celletti. Stability and chaos in celestial mechanics. Springer-Verlag, Berlin; published in association with Praxis Publishing Ltd., Chichester, 2010.

[78] Maciej J. Capiński. Covering relations and the existence of topologically normally hyperbolic invariant sets. Discrete Contin. Dyn. Syst., 23(3):705-725, 2009.

[79] Daniel Wilczak. Uniformly hyperbolic attractor of the Smale-Williams type for a Poincaré map in the Kuznetsov system. SIAM J. Appl. Dyn. Syst., 9(4):1263-1283, 2010. With online multimedia enhancements.

[80] Roberto Barrio, M. Angeles Martínez, Sergio Serrano, and Daniel Wilczak. When chaos meets hyperchaos: 4D Rössler model. Phys. Lett. A, 379(38):2300-2305, 2015.

[81] Gianni Arioli. Periodic orbits, symbolic dynamics and topological entropy for the restricted 3-body problem. Comm. Math. Phys., 231(1):1-24, 2002.

[82] Daniel Wilczak and Piotr Zgliczyński. Heteroclinic connections between periodic orbits in planar restricted circular three body problem. II. Comm. Math. Phys., 259(3):561-576, 2005.

[83] Maciej J. Capiński. Computer assisted existence proofs of Lyapunov orbits at $L_{2}$ and transversal intersections of invariant manifolds in the Jupiter-Sun PCR3BP. SIAM J. Appl. Dyn. Syst., 11(4):1723-1753, 2012.

[84] Maciej Capiński and Pablo Roldán. Existence of a center manifold in a practical domain around $L_{1}$ in the restricted three body problem. SIAM J. Appl. Dyn. Syst., 11(1):285-318, 2011.

[85] Gianni Arioli. Branches of periodic orbits for the planar restricted 3-body problem. Discrete Contin. Dyn. Syst., 11(4):745-755, 2004.

[86] Joseph Galante and Vadim Kaloshin. Destruction of invariant curves in the restricted circular planar three-body problem by using comparison of action. Duke Math. J., 159(2):275-327, 2011.

[87] Maciej J. Capiński and Piotr Zgliczyński. Transition tori in the planar restricted elliptic three-body problem. Nonlinearity, 24(5):1395-1432, 2011.

[88] Tomasz Kapela and Piotr Zgliczyński. The existence of simple choreographies for the $N$-body problem-a computerassisted proof. Nonlinearity, 16(6):1899-1918, 2003.

[89] Tomasz Kapela. $N$-body choreographies with a reflectional symmetry - computer assisted existence proofs. In EQUADIFF 2003, pages 999-1004. World Sci. Publ., Hackensack, NJ, 2005.

[90] Tomasz Kapela and Carles Simó. Computer assisted proofs for nonsymmetric planar choreographies and for stability of the Eight. Nonlinearity, 20(5):1241-1255, 2007.

[91] Kenneth R. Meyer, Glen R. Hall, and Dan Offin. Introduction to Hamiltonian dynamical systems and the N-body problem, volume 90 of Applied Mathematical Sciences. Springer, New York, second edition, 2009.

[92] C. L. Siegel and J. K. Moser. Lectures on celestial mechanics. Classics in Mathematics. Springer-Verlag, Berlin, 1995. Translated from the German by C. I. Kalme, Reprint of the 1971 translation.

[93] Jean-Philippe Lessard, Jason D. Mireles James and Julian Ransford, MATLAB codes available at http://archimede.mat.ulaval.ca/jplessard/AD/ 